\title{
Kinetics of oriented crystallization of polymers in the linear stress-orientation range in the series expansion approach
}

\author{
L. Jarecki ${ }^{*}$ R. B. Pecherski \\ Institute of Fundamental Technological Research, Polish Academy of Sciences, Pawinskiego 5B, 02-106 Warsaw, Poland
}

Received 11 September 2017; accepted in revised form 29 November 2017

\begin{abstract}
An analytical formula is derived for the oriented crystallization coefficient governing kinetics of oriented crystallization under uniaxial amorphous orientation in the entire temperature range. A series expansion approach is applied to the free energy of crystallization in the Hoffman-Lauritzen kinetic model of crystallization at accounting for the entropy of orientation of the amorphous chains. The series expansion coefficients are calculated for systems of Gaussian chains in linear stress-orientation range. Oriented crystallization rate functions are determined basing on the 'proportional expansion' approach proposed by Ziabicki in the steady-state limit. Crystallization kinetics controlled by separate predetermined and sporadic primary nucleation is considered, as well as the kinetics involving both nucleation mechanisms potentially present in oriented systems. The involvement of sporadic nucleation in the transformation kinetics is predicted to increase with increasing amorphous orientation. Example computations illustrate the dependence of the calculated functions on temperature and amorphous orientation, as well as qualitative agreement of the calculations with experimental results.
\end{abstract}

Keywords: modeling and simulation, kinetics of oriented crystallization, amorphous orientation, sporadic nucleation, predetermined nucleation

\section{Introduction}

Molecular deformation and orientation in the amorphous phase strongly accelerate crystallization of polymers. The orientation is caused by tensile stresses applied during processing. In polymer fluids, molecular orientation produced during melt spinning of fibers, film blowing, film casting, etc., is controlled by the deformation rate, or stress. Extension and orientation of chain macromolecules in polymer fluids under the deformation rate result in enhancement of tensile modulus and tenacity of the material, while the enhanced crystallization kinetics stabilizes polymer structure and properties. The tensile stresses and molecular orientation affect the dynamics of processing of crystallizing polymers and kinetics of structure formation. Besides high impact on the crystallization rate, the orientation influences thermodynamic behavior of the polymer and rises the equilibrium melting temperature.

In this work we focus on existing problems in modeling of crystallization kinetics under uniaxial molecular orientation, typical for fiber melt spinning processes. High values of the elongation rate in melt spinning processes result in significant amorphous orientation of the chain macromolecules. Dynamics of the processes is strongly coupled with fast crystallization induced by the orientation, in particular for slowly crystallizing polymers. Molecular orientation in the amorphous structure and the degree of crystallinity determine mechanical and physical properties of fibers. The role of orientation in the development of polymer structure and properties has been a subject of main interest on the fundamentals of melt spinning processes [1-7]. 
Experimental investigations on oriented crystallization have shown that the crystallization rate and equilibrium melting temperature increase with the amorphous orientation, and the transformation rate increases by orders of magnitude [8-15]. It has been shown that the effects are controlled by the entropy of deformation and orientation of chain macromolecules in the amorphous component [2,16-25]. Oriented crystallization during melt spinning strongly influences the process dynamic and axial profiles of fiber velocity, diameter, tensile stress, birefringence, amorphous and crystalline orientation, degree of crystallinity $[4,5,26,27]$. The increase in crystallization rate is estimated for several orders of the magnitude in fast melt spinning processes. Rapid oriented crystallization causes hardening of the polymer which prevents further drawing of the filaments on the spinning line and introduces limitation in the attenuation of the fiber and achievable spinning speed $[4,5,28]$. The influence of crystallization on the rheological behavior has been investigated experimentally, as well as by using modeling concepts [24, 29-40].

Significant effects of oriented crystallization has been indicated by modeling of melt spinning processes that include effects of hardening of the polymer by online crystallization, such as high-speed melt spinning [4, 5, 24, 28], melt blowing [41-43], pneumatic melt spinning in the Laval nozzle under supersonic air jet [43, 44].

In the modeling of polymer processing under variable molecular orientation and temperature conditions, the crystallization rate has been expressed by a quasi-static formula [5, 6] obtained by an extension of the Avrami-Evans formula basing on the nonisothermal Nakamura approach $[45,46]$. The Nakamura approach extended for oriented crystallization is considered as superior for predicting the development of crystallization [5]. The instantaneous crystallization rate reduces to a simple formula derived basing on an orientation- and temperature-dependent crystallization half-time in the quasi-static approximation, a directly observable kinetic characteristics of crystallization at fixed orientation and temperature $[46,47]$. The half-time analysis has been used by Spruiell and coworkers $[4,5]$ to discuss problems which appear in applying the experimental kinetics data in modeling of real melt spinning processes. Other mechanisms possible under variable molecular orientation and temperature, such as athermal nucleation [48-50] and memory effects [51-54] which result from transient distribution of cluster sizes [ 50 , $55]$ and transient molecular orientation $[56,57]$ have been indicated by Ziabicki and coworkers [46, 49, 55, 58-60] and Jarecki [61] basing on the AvramiEvans approach.

At large undercoolings, the nucleation mechanism may no longer be valid and a low temperature limit has been indicated for nucleation-controlled crystallization [55]. Then, the crystallization rate cannot be described by the nucleation-controlled mechanism. A failure of the crystallization half-time approach has been detected in the experimental investigations at the combination of large undercoolings and stresses in melt spinning, possibly due to a collapse of the kinetic model of crystal nucleation under such conditions [5, 62].

A phenomenological formula for the crystallization rate function has been proposed by Ziabicki $[2,47]$ basing on the analysis of the crystallization half-time of isothermal, oriented crystallization at constant molecular orientation. Series expansions of the rate function over the amorphous orientation factor has been considered in the analysis using the kinetic theory of nucleation and crystal growth with the effects of molecular orientation in the transformation free energy. In the phenomenological formula, the rate function increases exponentially with the square of the amorphous orientation factor multiplied by a positive phenomenological coefficient. The oriented crystallization coefficient has been estimated from independent experiments on pre-oriented PET fibers by Smith and Steward [9], Stein [10], Alfonso et al. [11] and its values increase with increasing crystallization temperature. Similar estimates of the coefficient and its temperature dependence have been provided by Spruiell $[4,5,27]$ from measurements of oriented crystallization rate during high-speed melt spinning of Nylon 6.

Several phenomenological formulas are provided for the temperature dependence of the oriented crystallization coefficient in the literature $[2,4,11]$ and all of them show inverse dependence on the undercooling. The formulas have been determined in the range of large undercoolings, and there is no reliable information, experimental or theoretical, from which the coefficient could be determined in the entire temperature range. Modeling of melt spinning processes with the oriented crystallization effects indicates that the oriented crystallization coefficient is responsible 
for important effects in the process dynamics and structure formation [4, 5, 28, 41-44]. None of the actually available models are satisfactory for calculating the kinetics of oriented crystallization under variable temperature in the entire range of undercooling with the use of the oriented crystallization coefficient proposed by Ziabicki [2, 47].

Another formula derived basing on the analysis of the crystallization half-time and the kinetic theory of crystallization [63] has been proposed by Spruiell and coworkers [7, 27]. A failure of the formula $[7,27]$ to predict effects of oriented crystallization in the modeling of fiber melt spinning may be due to a failure of the nucleation mechanism at combined large undercoolings and high orientations. Possibility of such a failure has been indicated by Ziabicki et al. [55] for large undercoolings and by George [62] for processes under high molecular orientation. It is indicated [55, 62] that under the extreme undercooling conditions combined with high amorphous orientation, the crystallization mechanism changes from nucleation-controlled one to a homogeneous, diffusion-controlled process throughout the melt with zero free energy barrier of nucleation. However, kinetics of oriented crystallization under such extreme conditions in fast melt spinning apparently has been adequately described by the phenomenological formula proposed by Ziabicki, with the use of quiescent kinetic data. Development of amorphous orientation is strongly coupled with the crystallization in processes under fast elongational flow. Under large tensile stresses, non-linear effects in the development of oriented amorphous structure vs. stress is predicted $[25,56$, $60,64,65]$. Closed-form analytical formula for nonlinear stress-orientation relation is derived for the entire range of tensile stresses, or orientations, basing on the inverse Langevin chain statistics which accounts for finite extensibility of the chain macromolecule in the amorphous rubbery network [66].

The formula for temperature dependence of the oriented crystallization coefficient is still needed to predict crystallization rate under large tensile stresses and fast cooling in the entire range of undercoolings. In the present paper, an analytical formula for the coefficient is derived basing on the kinetic model of crystallization [63] and experimental indications [5, 7, 27].

\section{Crystallization rate formulations}

Progress of crystallization is controlled by nucleation followed by crystal growth and its rate shows a maximum at an intermediate temperature between the equilibrium melting point and the glass transition temperature. Under non-isothermal conditions where the temperature changes over the range of variable crystallization rate the transformation progress is affected by the cooling rate. At faster cooling the crystallization time is shortened at each temperature interval and the progress of crystallization is suppressed. Molecular orientation produced under external orienting stresses is one of the processing factors that contributes to the thermodynamic driving force of crystallization and may significantly speed up the transformation rate. The orientation-induced enhancement of the crystallization kinetics applies to the entire crystallization temperature range and significantly modifies the influence of the cooling rate on crystallization at any processing. The orientation results in an earlier crystallization and enables crystallization in the case of slowly crystallizing polymers during fast cooling. In high-speed melt spinning processes the rates of online crystallization under molecular orientation increase by orders of magnitude and the enhancement in the transformation kinetics is higher at higher temperatures $[2,3,7]$.

Under isothermal conditions and zero amorphous orientation, the progress of crystallization is described by the Avrami equation $\theta(t)=1-\exp \left(-k t^{\mathrm{n}}\right)$ where $\theta(t)=X(t) / X_{\infty}$ is the conversion ratio by crystallization, $X(t)$ and $X_{\infty}$ are volume degrees of the transformation, the instantaneous one and at full conversion, respectively, $k$ the crystallization rate constant dependent on the temperature, $n$ the Avrami exponent.

The progress of non-isothermal crystallization is described by the Nakamura equation [45] $\theta(t)=$ $1-\exp \left[-\left(\int_{0}^{\mathrm{t}} K(T) \mathrm{d} t^{\prime}\right)^{\mathrm{n}}\right]$ at an isokinetic approximation where the temperature-dependent crystallization rate function $K(T)=[k(T)]^{1 / n}$. Under quiescent conditions, the rate function is described by the phenomenological formula [2]:

\section{$K(T)=K_{\max } \exp \left\lfloor-4 \ln 2\left(T-T_{\max }\right)^{2} / D^{2}\right\rfloor$.}

In our approach we follow the classical line similar in the form to the Kolmogoroff-Avrami-Evans theory in describing the crystallization kinetics, developed for the transformation under variable temperature and molecular orientation $[45,46]$. We account for the effects of variable amorphous orientation by deriving an analytical formula for the crystallization rate function $K\left(T, f_{\mathrm{a}}\right)$ basing on the 'proportional expansion' approach proposed by Ziabicki [46] for the 
crystallization kinetics under variable temperature and orientation.

We confine our considerations to constant mechanisms in the crystallization kinetics governed by thermal nucleation, sporadic and predetermined, and nucleation-controlled crystal growth in the quasi-static approximation. Athermal and memory effects are not accounted for. The quasi-static crystallization rate under variable temperature and orientation is expressed by the formula obtained by an extension of the Nakamura et al. [45] approach for oriented crystallization $[5,6]$ (Equation (1)):

$$
\frac{\mathrm{d} \theta}{\mathrm{d} t}=n K\left[T(t), f_{\mathrm{a}}(t)\right](1-\theta)[\ln (1-\theta)]^{-(1-\mathrm{n}) / \mathrm{n}}
$$

where $K$ is the crystallization rate function dependent on the actual temperature $T(t)$ and the actual orientation factor $f_{\mathrm{a}}(t)$. Equation (1) is equivalent to the formula obtained in the steady-state limit of a more general 'proportional expansion' approach [46] and is considered as a superior one for predicting the transformation rate in processes under variable temperature and orientation.

Equation(1) in the integral form reads (Equation (2)):

$\theta(t)=1-\exp \left\{-\left[\int_{0}^{\mathrm{t}} K\left(T(s), f_{\mathrm{a}}(s)\right) \mathrm{d} s\right]^{\mathrm{n}}\right\}$

where the kinetics and the degree of the transformation are determined by the temperature - and orientation - dependent rate function $K$ and its history.

Let us observe that in this discussion an analogy appears with the concept of the development of the micro-shear bands contribution in modeling of the deformation mechanisms in polymers and metals $[67,68]$.

The rate function in Equations (1) and (2) expresses by the inverse of the observable crystallization halftime, $K\left(T, f_{\mathrm{a}}\right)=(\ln 2)^{1 / \mathrm{n} / t_{1 / 2}}\left(T, f_{\mathrm{a}}\right)$, determined for quiescent crystallization at fixed temperature $T$ and orientation $f_{\mathrm{a}}[46,47]$. Constant Avrami exponent $n$ and constant mechanisms of nucleation-controlled crystallization are assumed. The rate function has been approximated by a phenomenological formula basing on the analysis of the crystallization half-time of isothermal crystallization at constant orientation by Ziabicki $[2,47]$. In the analysis, series expansions of the rate function over the factor $f_{\mathrm{a}}$ has been considered in the linear stress-orientation range basing on the kinetic theory of nucleation and crystal growth with the contribution of orientation to the crystallization free energy. The series expansion has been confined to the square term in $f_{\mathrm{a}}[2,47]$ (Equation (3)):

$K\left(T, f_{\mathrm{a}}\right)=K_{\max } \exp \left[-4 \ln 2 \frac{\left(T-T_{\max }\right)^{2}}{D^{2}}+A(T) f_{\mathrm{a}}^{2}\right]$

where the term linear in $f_{\mathrm{a}}$ had dropped out for symmetry reasons and the crystallization rate increases exponentially with $A(T) f_{\mathrm{a}}^{2}$. The global rate function has been considered in the series expansion approach and Equation (3) has been derived without considering the contributions of sporadic and predetermined nucleation, as well as crystal growth.

There is no reliable information from which the temperature dependence of the oriented crystallization coefficient $A$ could be determined for various polymers, except for some rough estimates from experimental investigations for a few polymers at large undercoolings. The magnitude of $A$ has been estimated to be in the range $10^{2}-10^{4}$ from independent experiments on the crystallization rate of pre-oriented PET samples, with the values increasing at increasing temperature $[9,11]$. Large values of $A$ indicate strong effects of amorphous orientation in the crystallization kinetics in real processes.

Large values of $A$ and their increase with temperature have been shown by Spruiell et al. [4, 5, 27] by online measurements of the crystallization rate during melt spinning of nylons. Although the phenomenological formula, Equation (3), has been derived basing on the series expansion in the linear stressorientation range, experimental investigations indicate validity of the formula also above the linear range, $f_{\mathrm{a}}>0.1$. In the present work we derive a closed-form formula for the coefficient $A(T)$ basing on fundamental formulations of the kinetic theory of crystal nucleation and growth [63]. The influence of sporadic and predetermined nucleation on $A(T)$ is accounted for.

The experimental information on the material function $A(T)$ still remains scarce, at the lack of any formula derived from the first principles. Large difficulties are associated with experiments designed to determine the rates of oriented crystallization at controlled amorphous orientation and temperature. One concludes from modeling of fast melt spinning processes that the function $A(T)$ is responsible for important effects in the processing dynamics and structure formation. For example, online solidification of 
the polymer by crystallization is predicted for slowly crystallizing polymers which significantly limits attenuation of the spun melt and the take-up velocity. Another formulation of the rate function $K\left(T, f_{\mathrm{a}}\right)$ proposed by Patel and coworkers [7, 27] bases on the Hoffman kinetic theory of crystallization [63] limited to the contribution of predetermined nucleation followed by crystal growth (Equation (4)):

$$
K\left(T, f_{\mathrm{a}}\right)=K(T .0) \exp \left[\frac{C_{3}}{T\left(T_{\mathrm{m}}^{0}-T\right)+C T^{2} f_{\mathrm{a}}^{2}}\right]
$$

where $K(T, 0)$ is the rate function at temperature $T$ and $f_{\mathrm{a}}=0, T_{\mathrm{m}}^{0}$ is the equilibrium melting temperature, $C_{3}$ material constant determined from isothermal crystallization rate, $C$ material constant estimated from online measurements of the crystallization rate.

An analysis of available approaches in modeling of melt spinning [5] has indicated that Equation (4) could not even qualitatively predict oriented crystallization rate at high spinning speeds, while it has predicted the rate at lower spinning speeds where no crystallization had been observed. It is claimed [5] that the failure of Equation (4) may result from the lack of nucleation mechanism in crystallization under very high orientation combined with large undercoolings. One has concluded in the analysis however, that the crystallization kinetics has been adequately described by Equation (3) with the phenomenological factor $\exp \left(A f_{\mathrm{a}}^{2}\right)$ also under the extreme conditions.

\section{Crystallization rate under variable orientation and temperature}

We calculate the rate functions for crystallization under variable temperature and orientation basing on the Hoffman-Lauritzen kinetic theory [63] accounting for sporadic and predetermined primary nucleation, as well as for crystal growth. Basing on the kinetic theory and the experimental indications $[5,7$, 27], analytical formulas for oriented crystallization coefficient $A(T)$ are derived in this paper. First, for oriented crystallization involving separate sporadic and predetermined nucleation, and next for processes with both types of primary nucleation present in the system. Our calculation are based on the 'proportional expansion' approach proposed by Ziabicki [46] in the steady-state limit.

The function $K\left(T, f_{\mathrm{a}}\right)$ for process under variable temperature $T(t)$ and orientation $f_{\mathrm{a}}(t)$ is inversely proportional to the instantaneous crystallization half-time (Equation (5)):
$K\left(T, f_{\mathrm{a}}\right)=\frac{(\ln 2)^{1 / \mathrm{n}}}{t_{1 / 2}\left(T, f_{\mathrm{a}}\right)}$

For processes with isolated sporadic nucleation and for processes with predetermined nucleation (heterogeneous, memory nuclei) the rate functions read, as shown in Equations (6) and (7) respectively [46]:

$$
\begin{aligned}
& K\left(T, f_{\mathrm{a}}\right)=\left[\frac{1}{n} \dot{N}\left(T, f_{\mathrm{a}}\right) R_{0}^{4-\mathrm{n}} G^{\mathrm{n}-1}\left(T, f_{\mathrm{a}}\right)\right]^{1 / n} \\
& K\left(T, f_{\mathrm{a}}\right)=\left(N_{0} R_{0}^{3-n}\right)^{1 / \mathrm{n}} G\left(T, f_{\mathrm{a}}\right)
\end{aligned}
$$

where $\dot{N}\left(T, f_{\mathrm{a}}\right)$ is the steady-state homogeneous nucleation rate, $N_{0}$ the number of predetermined nuclei per unit volume, $G\left(T, f_{\mathrm{a}}\right)$ linear growth rate at $T$ and $f_{\mathrm{a}}, n$ Avrami exponent, $R_{0}$ dimension of the crystallite in which the growth does not proceed.

From the kinetic model of Hoffman-Lauritzen, the sporadic nucleation and crystal growth rates read as shown in Equations (8) and (9):

$$
\begin{aligned}
& \dot{N}\left(T, f_{\mathrm{a}}\right)=N_{\mathrm{s}} \frac{k T}{h} \exp \left\{-\frac{U^{*}}{R\left(T-T_{\infty}\right)}-\frac{2 c^{2} \sigma^{2} \sigma_{\mathrm{e}}}{k T\left[\Delta g\left(T, f_{\mathrm{a}}\right)\right]^{2}}\right\} \\
& G\left(T, f_{\mathrm{a}}\right)=G_{0} \exp \left\{-\frac{U^{*}}{R\left(T-T_{\infty}\right)}+\frac{4 b \sigma \sigma_{\mathrm{e}}}{k T \Delta g\left(T, f_{\mathrm{a}}\right)}\right\}
\end{aligned}
$$

where $\Delta g\left(T, f_{\mathrm{a}}\right)$ is free energy of crystallization per unit volume of the crystalline phase at $T$ and $f_{\mathrm{a}}, N_{\mathrm{s}}$ the number of single kinetic units per unit volume of the amorphous phase identified with statistical segments of the chains, $k T / h$ thermal frequency, $U^{*}$ activation energy of molecular transport, $T_{\mathrm{m}}$ temperature at which molecular motions cease near the crystal surface, $\sigma_{\mathrm{e}}$ and $\sigma$ free energy densities at the end and side surfaces, $b$ thickness of the surface nucleus, $c$ constant dependent on the nucleus geometry, $h$ and $R$ are the Planck and gas constants. The preexponential factor $G_{0}$ is proportional to thermal frequency.

The tensile stresses determine strain energy and contribute to the enthalpy and entropy of the amorphous and crystalline phases. The thermodynamic functions of the phases modified by the orientation $f_{\mathrm{a}}$ at temperature $T$ read:

- for the enthalpy and entropy of the amorphous phase in Equations (10a) and (10b):

$h_{\mathrm{a}}\left(T, f_{\mathrm{a}}\right)=h_{\mathrm{a}}^{0}(T)+\delta h_{\mathrm{a}}\left(T, f_{\mathrm{a}}\right)$ 
$s_{\mathrm{a}}\left(T, f_{\mathrm{a}}\right)=s_{\mathrm{a}}^{0}(T)+\delta s_{\mathrm{a}}\left(T, f_{\mathrm{a}}\right)$

- and for the crystalline phase in Equations (11a) and (11b):

$h_{\mathrm{c}}\left(T, f_{\mathrm{a}}\right)=h_{\mathrm{c}}^{0}(T)+\delta h_{\mathrm{c}}\left(T, f_{\mathrm{a}}\right)$

$s_{\mathrm{c}}\left(T, f_{\mathrm{a}}\right)=s_{\mathrm{c}}^{0}(T)+\delta s_{\mathrm{c}}\left(T, f_{\mathrm{a}}\right)$

where $\delta h_{\mathrm{a}}, \delta s_{\mathrm{a}}, \delta h_{\mathrm{c}}, \delta s_{\mathrm{c}}$ are the contribution of the orientation to the thermodynamic functions at temperature $T$ and $h_{\mathrm{a}}^{0}, s_{\mathrm{a}}^{0}, h_{\mathrm{c}}^{0}, s_{\mathrm{c}}^{0}$ are the functions at zero orientation and $T$.

In general, the contributions of orientation to the thermodynamic functions modify the crystallization free energy density (Equation (12)):

$$
\begin{aligned}
\Delta g\left(T, f_{\mathrm{a}}\right) & =\Delta g^{0}(T)+\delta h_{\mathrm{c}}\left(T, f_{\mathrm{a}}\right)-\delta h_{\mathrm{a}}\left(T, f_{\mathrm{a}}\right)- \\
& -T\left[\delta s_{\mathrm{c}}\left(T, f_{\mathrm{a}}\right)-\delta s_{\mathrm{a}}\left(T, f_{\mathrm{a}}\right)\right]
\end{aligned}
$$

where $\Delta g^{0}(T)=\Delta h^{0}(T)-T \Delta s^{0}(T)$ and $\Delta h^{0}, \Delta s^{0}$ are the enthalpy and entropy of crystallization in the unoriented state. The entropy of amorphous phase decreases faster than the entropy of the crystalline phase with decreasing $T$. The decrease in the entropies at decreasing temperature is accounted for in the entropy of crystallization of the unoriented polymer by a reducing factor $f(T) \cong 2 T /\left(T+T_{\mathrm{m}}^{0}\right)$, the same as for the decrease in the crystallization enthalpy, and reads $\Delta s^{0}(T)=f(T) \Delta h_{\mathrm{m}} / T_{\mathrm{m}}^{0}$ where $T_{\mathrm{m}}^{0}$ and $\Delta h_{\mathrm{m}}$ are the equilibrium melting point and the melting heat, respectively. With the reducing factor the crystallization free energy in the unoriented state reads $\Delta g^{0}(T)=f(T) \Delta h_{\mathrm{m}} \Delta T / T_{\mathrm{m}}^{0}$ where $\Delta T=T_{\mathrm{m}}^{0}-T$ is the undercooling [63].

In oriented crystallization, the largest contribution of orienting stresses to the crystallization free energy $\Delta g\left(T, f_{\mathrm{a}}\right)$ concerns entropy of orientation of the amorphous phase, $\delta s_{\mathrm{a}}$, while the remaining contributions $\delta s_{\mathrm{c}}, \delta h_{\mathrm{a}}$ and $\delta h_{\mathrm{c}}$ are considered as relatively small and usually are neglected $[25,69,70]$. We neglect the temperature effects in the entropy contribution $\delta s_{\mathrm{a}}$, in comparison with large contribution of the configurational entropy of the chains under orienting stresses, and the contribution is approximated by the change in configurational entropy under orientation, $\delta s_{\mathrm{a}}\left(T, f_{\mathrm{a}}\right) \cong \delta s_{\mathrm{a}}^{\operatorname{conf}}\left(f_{\mathrm{a}}\right)<0$. The approximation is confirmed by entropic elasticity of the flexible chain amorphous polymers determined by configurational entropy of the chains.
With the orientation effects in the crystallization driving force approximated by the change in configurational entropy of amorphous chains, free energy density of crystallization is enhanced by amorphous orientation and reads as shown in Equation (13):

$\Delta g\left(T, f_{\mathrm{a}}\right)=\Delta g^{0}(T)+T \delta s_{\mathrm{a}}^{\text {conf }}\left(f_{\mathrm{a}}\right)$

The orientation entropy contributes negative term to the transformation free energy and considerably speeds up the crystallization. The equilibrium melting point also increases with increasing orientation according to Equation (14):

$T_{\mathrm{m}}\left(f_{\mathrm{a}}\right)=\frac{T_{\mathrm{m}}^{0}}{1-\frac{\delta s_{\mathrm{a}}^{\text {conf }}\left(f_{\mathrm{a}}\right)}{\Delta s^{0}(T)}} \cong T_{\mathrm{m}}^{0}\left[1+\frac{\delta s_{\mathrm{a}}^{\text {conf }}\left(f_{\mathrm{a}}\right)}{\Delta s^{0}(T)}\right](14)$

where $\delta s_{\mathrm{a}}^{\text {conf }}\left(f_{\mathrm{a}}\right) / \Delta s^{0}(T)>0$. The increase in the equilibrium melting point under orientation results in an increase of the undercooling by about $T_{\mathrm{m}}^{0} \delta s_{\mathrm{a}}^{\operatorname{conf}}\left(f_{\mathrm{a}}\right) / \Delta s^{0}(T)$, without changing the temperature. With the thermodynamic effect of orientation, the nucleation rates of sporadic nucleation and crystal growth increase exponentially due to reduction of the thermodynamic barriers of nucleation, Equations (8) and (9).

A more detailed analysis of the other contributions of orienting stresses to the crystallization free energy including enthalpy of the amorphous chains and the crystals strain energy shows that all these effects, neglected in the present approach, contribute to higher undercooling, higher crystallization driving force and faster nucleation rates $[23,25,69,70]$.

\section{The series expansion approach}

The change in the configurational entropy of the amorphous chains under the orientation is considered as sufficiently smooth function of the Hermans axial orientation factor $f_{\mathrm{a}}$ and is expanded in the power series over $f_{\mathrm{a}}$ around zero orientation, $f_{\mathrm{a}}=0$. The series expansion reads as shown by Equation (15) $[2,47]$ :

$\delta s_{\mathrm{a}}^{\mathrm{conf}}\left(f_{\mathrm{a}}\right)=-k\left(a_{2} f_{\mathrm{a}}^{2}+a_{3} f_{\mathrm{a}}^{3}+\ldots\right)$

where the zero-order and linear terms drops out because of physical and geometrical reasons [2, 47], i.e. the entropy of the amorphous phase in oriented state cannot exceed entropy of the unoriented phase at positive or negative $f_{\mathrm{a}}$. With the series expansion, the rates of sporadic nucleation and crystal growth read as shown by Equation (16) and (17): 


$$
\begin{aligned}
& \dot{N}\left(T, f_{\mathrm{a}}\right)=\dot{N}\left(T, f_{\mathrm{a}}=0\right) \exp \left[\frac{4 c^{2} \sigma^{2} \sigma_{\mathrm{e}}}{\left(f \Delta h_{\mathrm{m}}\right)^{3}}\left(\frac{T_{\mathrm{m}}^{0}}{\Delta T}\right)^{3}\left(a_{2} f_{\mathrm{a}}^{2}+a_{3} f_{\mathrm{a}}^{3}+\ldots\right)\right] \\
& G\left(T, f_{\mathrm{a}}\right)=G\left(T, f_{\mathrm{a}}=0\right) \exp \left[\frac{4 b \sigma \sigma_{\mathrm{e}}}{\left(f \Delta h_{\mathrm{m}}\right)^{2}}\left(\frac{T_{\mathrm{m}}^{0}}{\Delta T}\right)^{2}\left(a_{2} f_{\mathrm{a}}^{2}+a_{3} f_{\mathrm{a}}^{3}+\ldots\right)\right]
\end{aligned}
$$

with the lowest term quadratic in $f_{\mathrm{a}}$ in the exponential functions responsible for the orientation effects.

In the series expansion approach, the rate function of oriented crystallization controlled solely by sporadic nucleation reads as shown by Equation (18):

$$
K_{\mathrm{s}}\left(T, f_{\mathrm{a}}\right)=K_{\mathrm{s}}^{0}(T) \exp \left[A_{\mathrm{s}}(T) f_{\mathrm{a}}^{2}+B_{\mathrm{s}}(T) f_{\mathrm{a}}^{3}+\ldots\right]
$$

where the temperature-dependent oriented crystallization coefficients are expressed by Equations (19a) and (19b):

$A_{\mathrm{s}}(T)=\frac{4 b \sigma \sigma_{\mathrm{e}}}{\left(f \Delta h_{\mathrm{m}}\right)^{2}}\left(\frac{T_{\mathrm{m}}^{0}}{\Delta T}\right)^{2}\left(\frac{1}{n} \frac{c^{2} \sigma}{b f \Delta h_{\mathrm{m}}} \frac{T_{\mathrm{m}}^{0}}{\Delta T}+\frac{n-1}{n}\right) a_{2}$

$B_{\mathrm{s}}(T)=\frac{a_{3}}{a_{2}} A_{\mathrm{s}}(T)$

For process controlled solely by predetermined nucleation we have Equations (20) and (21):

$$
\begin{aligned}
& K_{\mathrm{p}}\left(T, f_{\mathrm{a}}\right)=K_{\mathrm{p}}^{0}(T) \exp \left[A_{\mathrm{p}}(T) f_{\mathrm{a}}^{2}+B_{\mathrm{p}}(T) f_{\mathrm{a}}^{3}+\ldots\right] \\
& A_{\mathrm{p}}(T)=\frac{4 b \sigma \sigma_{\mathrm{e}}}{\left(f \Delta h_{\mathrm{m}}\right)^{2}}\left(\frac{T_{\mathrm{m}}^{0}}{\Delta T}\right)^{2} a_{2} \\
& B_{\mathrm{p}}(T)=\frac{a_{3}}{a_{2}} A_{\mathrm{p}}(T)
\end{aligned}
$$

The pre-exponential factors $K_{\mathrm{s}}^{0}(T)$ and $K_{\mathrm{p}}^{0}(T)$ in Equations (18) and (20) are the rate functions at $f_{\mathrm{a}}=0$.

\section{The linear stress-orientation conditions}

Extension and orientation of chains along the tensile stress axis affects thermodynamics and kinetics of crystallization. Due to exponential stretch relaxation present in polymeric fluids, orientational effectivity of uniaxial elongational flow is determined by the product of the flow elongation rate and the stretch relaxation time, $\dot{q} \tau$, while the effectivity of the processing time is controlled by the reduced time, $t / \tau$. The same behavior is shown by the configurational entropy of the chains and the elastic tensile stresses in such systems $[2,25,60,66]$. Modeling of timeevolution of molecular deformation of the chains in polymer melts under uniaxial elongational flow is based on the evolution equations (Equations (22a) and (22b)) for the average chain extension coefficients $\lambda$ and $\lambda_{\perp}$ along and perpendicular to the flow axis $[60,66]$ :

$\frac{\mathrm{d} \lambda^{2}}{\mathrm{~d}(t / \tau)}+[E(t)-2 \dot{q} \tau] \lambda^{2}-1=0$

$$
\frac{\mathrm{d} \lambda_{\perp}^{2}}{\mathrm{~d}(t / \tau)}+[E(t)+\dot{q} \tau] \lambda_{\perp}^{2}-1=0
$$

where $E(t)$ is the Peterlin non-linear modulus of elasticity which varies in time between unity in the linear Gaussian range and infinity at full chain extension. The amorphous orientation factor $f_{\mathrm{a}}$ and the average tensile stresses $\Delta p$ have been calculated in $[60,66]$ basing on a non-linear inverse-Langevin chain statistics in systems subjected to uniaxial elongational flow with the molecular stretch relaxation accounted for. The calculated stress-orientation relations coincide into a unique, single master plot in the entire range of the elongation rates, independent of the elongation rate and the relaxation time because the tensile stress and orientation undergo the stretch relaxation simultaneously. The unique stress-orientation relations behavior is a consequence of the fact that the molecular orientation is controlled solely by the stress.

The stress-orientation formula shows linearity in the Gaussian range of chain extensions which produce orientation in the range of $f_{\mathrm{a}}$ between zero and of about 0.1 . In the linear range, the average molecular elongation coefficients, with the stretch relaxation accounted for, follow the relation $\lambda(t) \lambda_{\perp}^{2}(t) \cong 1$ during the processing time $[60,66]$. The average tensile stress in the linear range $\langle\Delta p\rangle(t)=v k T\left\lfloor\lambda^{2}(t)-1 / \lambda(t)\right\rfloor$ and the orientation factor $f_{\mathrm{a}}(t)=\left\lfloor\lambda^{2}(t)-1 / \lambda(t)\right\rfloor /(5 N)$ where $v$ is the number of elastic chains per unit volume, $N$ the number of statistical segments per chain. At higher tensile stresses, or orientations, present for example in high-speed melt spinning of fibers, nonlinear inverse Langevin stress-orientation behavior also does not show up explicitly the molecular stretch 
relaxation which is hidden in the master stress-orientation plot $[60,66]$.

In drawing deformation of solid elastic amorphous polymers, molecular deformation and orientation are controlled by the draw ratio, not by the elongation rate or stress, and the contribution of the molecular stretch relaxation is strongly limited $[2,65]$. Crosslinked solids in rubbery state also show the linear Gaussian range in the stress-orientation behavior and an abrupt increase in the stress due to a limited extensibility of the network chains above the linear range, similar to the master plot determined for polymer melts. The difference with the flow deformation of polymeric melts is that the molecular elongation coefficients in the rubbery solids coincide with the macroscopic elongation coefficients. For isochoric uniaxial drawing of a rubbery cross-linked solid the relation $\lambda \lambda_{\perp}^{2}=1$ also holds in the linear stress-orientation range [71].

We determine the series expansion coefficients in Equation (15) for systems of flexible linear chains subjected to uniaxial tensile stresses in the linear stress-orientation range under uniaxial elongational flow or uniaxial elongation of rubbery solids. The average entropy of deformation in the Gaussian range per unit volume of the amorphous phase approximated by the change in configurational entropy of the chains $[8,19,60] \delta S_{\mathrm{a}}^{\text {conf }}=v\left\langle\delta S_{\mathrm{a}}^{\text {conf }}\right\rangle=$ $-v k\left(\lambda^{2}+2 / \lambda-3\right) / 2$ where $\left\langle\delta S_{\mathrm{a}}^{\text {conf }}\right\rangle$ is an average decrease in the configurational entropy per single chain.

The series expansions of the deformation entropy and the orientation factor over the powers of $\lambda-1$ read as shown by Equations (23) and (24):

$$
\begin{aligned}
& \delta s_{\mathrm{a}}^{\mathrm{conf}}=-\frac{\nu k}{2}\left[3(\lambda-1)^{2}-2(\lambda-1)^{3}+\ldots\right] \\
& f_{\mathrm{a}}=\frac{1}{5 N}\left[3(\lambda-1)+(\lambda-1)^{3}+\ldots\right]
\end{aligned}
$$

where the zero- and first-order terms in the series expansion of $\delta s_{\mathrm{a}}^{\text {conf }}$ and the zero- and second-order terms in the expansion of $f_{\mathrm{a}}$ drop out.

The inverse series expansion applied for Equation (24) reads (Equation (25)):

$$
A_{\mathrm{s}}(T)=\frac{50 b \sigma \sigma_{\mathrm{e}}}{3\left(f \Delta h_{\mathrm{m}}\right)^{2}}\left(\frac{T_{\mathrm{m}}^{0}}{\Delta T}\right)^{2}\left(\frac{1}{n} \frac{c^{2} \sigma}{b f \Delta h_{\mathrm{m}}} \frac{T_{\mathrm{m}}^{0}}{\Delta T}+\frac{n-1}{n}\right) \frac{\rho_{\mathrm{c}} N_{\mathrm{A}} n_{\mathrm{b}}^{2} M_{\mathrm{e}}}{C_{\infty}^{2} m^{2}}
$$

where the second-order term drops out. With Equation (25), the decrease in the configurational entropy per unit volume caused by the orienting stresses is expressed by the series expansion over $f_{\mathrm{a}}$ and is given by Equation (26):

$$
\begin{aligned}
\delta s_{\mathrm{a}}^{\mathrm{conf}} & =-\frac{3 v k}{2}\left(\frac{5 N}{3}\right)^{2}\left(f_{\mathrm{a}}^{2}-\frac{10 N}{9} f_{\mathrm{a}}^{3}+\ldots\right)= \\
& =-k\left(a_{2} f_{\mathrm{a}}^{2}+a_{3} f_{\mathrm{a}}^{3}+\ldots\right)
\end{aligned}
$$

The number of statistical segments in the linear chain undergoing elastic deformation is $N=n_{\mathrm{b}} M /\left(C_{\infty} m\right)$ where $M$ is the chain molecular weight, $n_{\mathrm{b}}$ number of rigid bonds in the chemical unit of the chain, $m$ molecular weight of the chemical unit, $C_{\infty}$ the characteristic ratio. We assume that the elastic chains subjected to the deformation are identified with the chains between the entanglements with the entanglement molecular weight $M=M_{\mathrm{e}}$. Then, the number of elastic chains per a volume transforming to a unit volume of the crystalline phase $v=\rho_{\mathrm{c}} N_{\mathrm{A}} / M_{\mathrm{e}}$ where $\rho_{\mathrm{c}}$ is density of the crystalline phase, $N_{\mathrm{A}}$ the Avogadro number and the coefficients are given by Equation (27):

$a_{2}=\frac{25}{6} \frac{\rho_{\mathrm{c}} N_{\mathrm{A}} n_{\mathrm{b}}^{2} M_{\mathrm{e}}}{C_{\infty}^{2} m^{2}}, \quad a_{3}=-\frac{10}{9} \frac{n_{\mathrm{b}} M_{\mathrm{e}}}{C_{\infty} m} a_{2}$

\section{Discussion}

With the aim of deriving the first oriented crystallization coefficient $A(T)$, we consider the kinetics of oriented crystallization in real systems as a process involving independent sporadic and predetermined nucleation followed by crystal growth. First, we derive the coefficients for processes with separate nucleation mechanisms, sporadic and predetermined, and next for processes involving both types of nucleation.

\subsection{Oriented crystallization coefficients at separated sporadic and predetermined nucleation}

With the expansion coefficients $a_{2}, a_{3}$ given by Equation (27), the coefficients for processes with separated sporadic nucleation read as shown by Equations (28a) and (28b): $\lambda=1+\frac{5 N}{3} f_{\mathrm{a}}-\frac{1}{3}\left(\frac{5 N}{3}\right)^{3} f_{\mathrm{a}}^{3}+\ldots$ 


$$
B_{\mathrm{s}}(T)=-\frac{10}{9} \frac{n_{\mathrm{b}} M_{\mathrm{e}}}{C_{\infty} m} A_{\mathrm{s}}(T)
$$

and for processes with separated predetermined nucleation by Equations (29a) and (29b):

$$
\begin{aligned}
& A_{\mathrm{p}}(T)=\frac{50 b \sigma \sigma_{\mathrm{e}}}{3\left(f \Delta h_{\mathrm{m}}\right)^{2}}\left(\frac{T_{\mathrm{m}}^{0}}{\Delta T}\right)^{2} \frac{\rho_{\mathrm{c}} N_{\mathrm{A}} n_{\mathrm{b}}^{2} M_{\mathrm{e}}}{C_{\infty}^{2} m^{2}} \\
& B_{\mathrm{p}}(T)=-\frac{10}{9} \frac{n_{\mathrm{b}} M_{\mathrm{e}}}{C_{\infty} m} A_{\mathrm{p}}(T)
\end{aligned}
$$

As an example, we compute the coefficient $A_{\mathrm{p}}(T)$ vs. temperature for processes with predetermined nucleation and the ratio $A_{\mathrm{s}}(T) / A_{\mathrm{p}}(T)$ of the coefficients for sporadic and predetermined nucleation for isotactic polypropylene (iPP), polyethylene terephthalate (PET), Nylon 66 and poly(L-lactide) (PLLA) using material parameters listed in Table 1. Large positive values of $A_{\mathrm{p}}$ are predicted in the computations. Figure 1 shows $A_{\mathrm{p}}$ plotted vs. undercooling $\Delta T=T_{\mathrm{m}}^{0}-T$. Values of $A_{\mathrm{p}}$ increase from of about several hundred at large $\Delta T$ to several thousand at decreasing $\Delta T$ to $30 \mathrm{~K}$ in the case of PET, Nylon 66 and PLLA. For iPP the coefficient is higher by a factor of about two in the entire range of $\Delta T$. All plots show steep increase of $A_{\mathrm{p}}$ tending to infinity at approaching the equilibrium melting temperature $T_{\mathrm{m}}^{0}$. Larger values of $A_{\mathrm{p}}$ for iPP result from low melting heat at which contribution of the configurational entropy of the chain deformation to the crystallization free energy is higher, relative to the crystallization enthalpy. The Avrami exponent does not influence $A_{\mathrm{p}}$.

Experimental points in Figure 1 show the values of the oriented crystallization coefficient A determined for PET at large undercoolings [11]. There is lack

Table 1. Material parameters used in the computations

\begin{tabular}{|ll|c|c|c|c|}
\hline \multicolumn{2}{|c|}{ Parameter } & iPP & PET & Nylon 66 & PLLA \\
\hline$T_{\mathrm{m}}^{0}$ & {$[\mathrm{~K}]$} & 453 & 553 & 537 & 448 \\
\hline$\Delta h_{\mathrm{m}}$ & {$[\mathrm{J} / \mathrm{g}]$} & 165.0 & 123.7 & 188.3 & 93.1 \\
\hline$\rho_{0}$ & {$\left[\mathrm{~g} / \mathrm{cm}^{3}\right]$} & 1.145 & 1.356 & 1.122 & 0.776 \\
\hline$\rho_{1}$ & {$\left[\mathrm{~g} /\left(\mathrm{cm}^{3} \cdot \mathrm{K}\right)\right]$} & $9.03 \cdot 10^{-4}$ & $5.00 \cdot 10^{-4}$ & $5.45 \cdot 10^{-4}$ & $4.62 \cdot 10^{-4}$ \\
\hline$\rho_{\mathrm{c}}$ & {$\left[\mathrm{g} / \mathrm{cm}^{3}\right]$} & 0.938 & 1.457 & 1.24 & 1.37 \\
\hline$\sigma$ & {$\left[\mathrm{J} / \mathrm{cm}^{2}\right]$} & $1.15 \cdot 10^{-6}$ & $1.09 \cdot 10^{-6}$ & $1.07 \cdot 10^{-6}$ & $1.20 \cdot 10^{-6}$ \\
\hline$\sigma_{\mathrm{e}}$ & {$\left[\mathrm{J} / \mathrm{cm}^{2}\right]$} & $6.23 \cdot 10^{-6}$ & $9.09 \cdot 10^{-6}$ & $11.8 \cdot 10^{-6}$ & $6.26 \cdot 10^{-6}$ \\
\hline$b$ & {$[\mathrm{~cm}]$} & $6.26 \cdot 10^{-8}$ & $5.95 \cdot 10^{-8}$ & $3.7 \cdot 10^{-8}$ & $5.17 \cdot 10^{-8}$ \\
\hline$m$ & {$\left[\mathrm{~g} / \mathrm{mole}^{2}\right]$} & 42 & 192 & 226 & 72 \\
\hline$n_{\mathrm{b}}$ & & 2 & 6 & 14 & 3 \\
\hline$C_{\infty}$ & & 5.7 & 4.0 & 5.9 & 10.5 \\
\hline$M_{\mathrm{e}}$ & 6900 & 1630 & 2010 & 8000 \\
\hline
\end{tabular}

of experimental data at lower undercoolings due to difficulties in conducting experiments on oriented crystallization at very high rates under lower undercoolings. The values of the kinetic coefficient A shown by the points in Figure 1 have been determined in [11] for PET preoriented amorphous fibers obtained by melt spinning at several take-up speeds. The oriented crystallization rate was measured for samples characterized by several values of the amorphous orientation factor, crystallized isothermally at several temperatures well below the temperature of maximum crystallization rate and quenched after an appropriate time below the glass transition temperature $T_{\mathrm{g}}$. In determining the orientation factor, form birefringence was neglected on the assumption that no voids are present in the fibers melt-spun at low and moderately high speeds [11]. Crystallization rate was determined for samples tightly wound on a metal frame to avoid relaxation of the amorphous orientation. Also low temperature range chosen for the isothermal crystallization, terminated by quenching which ensured slow enough non-isothermal crystallization at the quenching, indicates high enough credibility of the kinetic data. Higher rates of oriented crystallization at higher temperatures unable adequate control of crystallization temperature, orientation and the transformation rate for obtaining credible kinetic data.

The experimental points of the oriented crystallization coefficient $A$ in Figure 1 are of the order of the coefficient $A_{\mathrm{p}}$ (several hundreds) calculated for PET

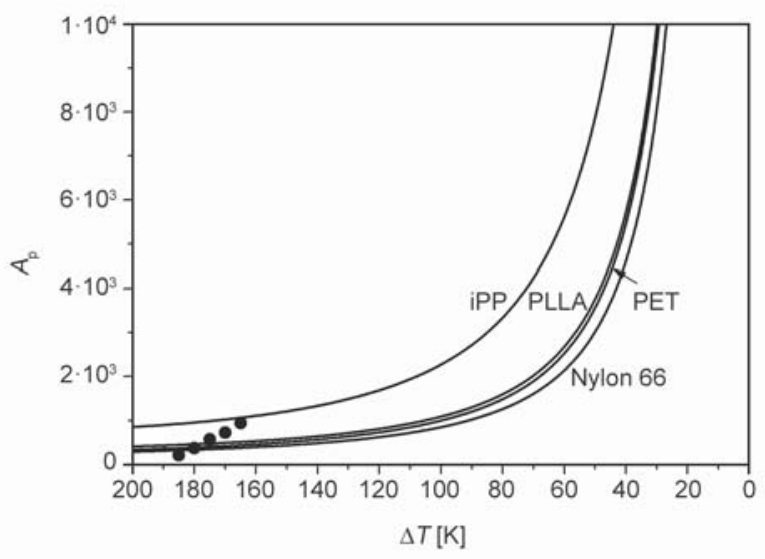

Figure 1. Oriented crystallization coefficient $A_{\mathrm{p}}$ for processes with separate predetermined nucleation vs. undercooling $\Delta T=T_{\mathrm{m}}^{0}-T$ computed for iPP, PET, Nylon 66 and PLLA from Equation (29a). Experimental points for PET obtained on isothermal crystallization of as-spun amorphous PET fibers with various amorphous orientation [11]. 


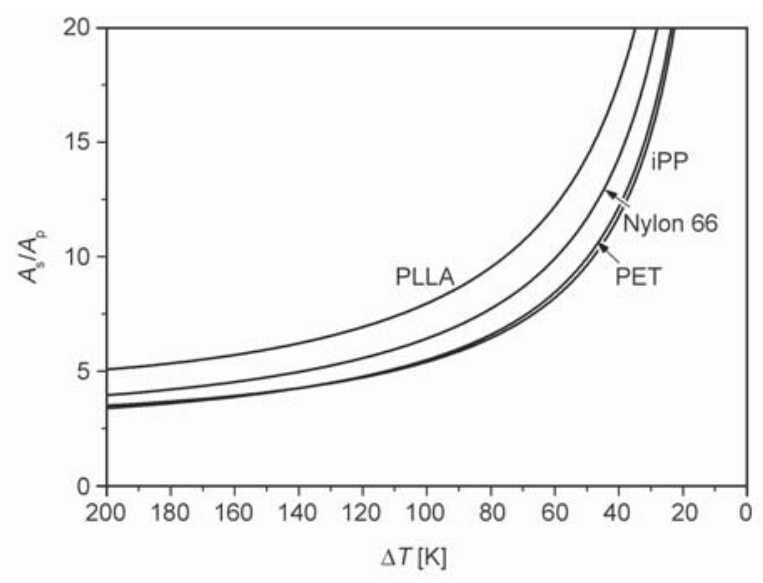

Figure 2. The ratio of the oriented crystallization coefficients $A_{\mathrm{s}} / A_{\mathrm{p}}$ for processes with separated sporadic and predetermined nucleation vs. undercooling $\Delta T=T_{\mathrm{m}}^{0}-T$ computed from Equations (28a) and (29a) for iPP, PET, Nylon 66 and PLLA at $n=2$

from Equations (29a) for crystallization controlled by predetermined nucleation and also increase with decreasing the undercooling $\Delta T$. In the range of rather low values of the factor $f_{\mathrm{a}}$ of the experimental samples we assign higher credibility to the coefficient $A_{\mathrm{p}}$ plotted in Figure 1, rather than to plotting the coefficient $A_{\mathrm{S}}$ for sporadic nucleation. Higher experimental values of $A$ than the calculated values in Figure 1 for $A_{\mathrm{p}}$ at lower undercoolings $\Delta T$ may result from a contribution of an additional crystallization possible at quenching from higher temperatures, accounted as the isothermal crystallization.

Figure 2 shows the plots of the ratio $A_{\mathrm{s}} / A_{\mathrm{p}}$ of the oriented crystallization coefficients computed for iPP, PET, Nylon 66 and PLLA from Equations (28a) and (29a) for processes with sporadic and predetermined nucleation as functions of undercooling $\Delta T$ at Avrami exponent $n=2$. The plots show that the oriented crystallization coefficient for sporadic primary nucleation is higher by a factor of about 4 up to 7 at larger undercoolings. At decreasing the undercooling, the ratio of the coefficients increases to higher values and tends to infinity at approaching $T_{\mathrm{m}}^{0}$. Larger values of $A_{\mathrm{s}}$ are a consequence of higher impact of the orientation entropy on the free energy barrier of sporadic nucleation than on the barrier of crystal growth. The influence of orientation on the sporadic nucleation free energy barrier and, in consequence, on the coefficient $A_{\mathrm{s}}$ is predicted to be lower at higher Avrami exponents $n$. Figure 3 illustrate low influence of $n$ at large undercoolings at which the $A_{\mathrm{s}} / A_{\mathrm{p}}$ ratio decreases by a factor of about 1-2 at increasing $n$ from 2 to 4 . The decrease is larger at low undercoolings.
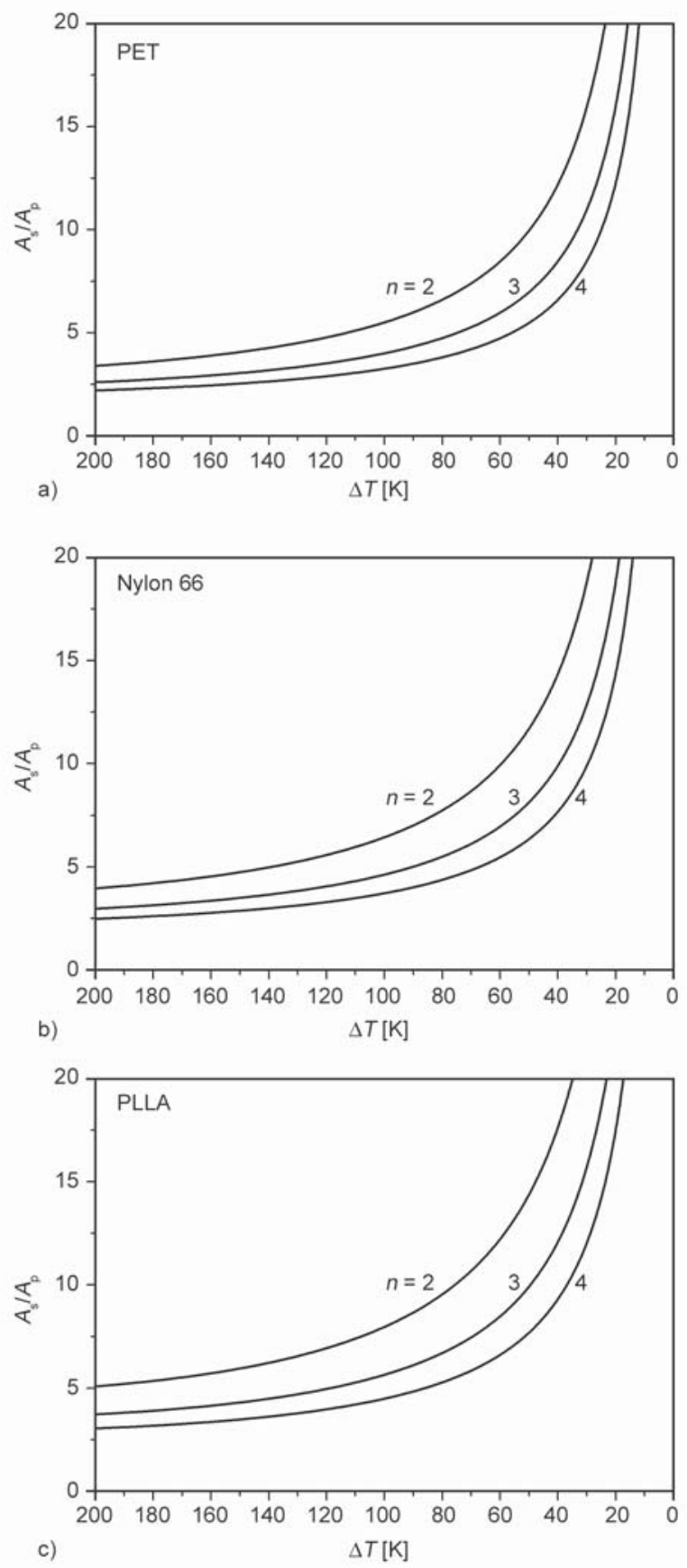

Figure 3. Ratio $A_{\mathrm{s}} / A_{\mathrm{p}}$ of the oriented crystallization coefficients vs. undercooling $\Delta T=T_{\mathrm{m}}^{0}-T$ computed from Equations (28a) and (29a) for PET (a), Nylon 66 (b) and PLLA (c) at $n=2,3,4$

No significant difference is predicted for the tested polymers.

\subsection{Rate functions at separate nucleation mechanisms}

Figures 4 show the plots of reduced rate function $K_{\mathrm{p}}\left(T, f_{\mathrm{a}}\right) / K_{\mathrm{p}}^{0}(T)$ computed from Equation (20) and $(29 \mathrm{a}, \mathrm{b})$ vs. amorphous orientation $f_{\mathrm{a}}^{2}$ for processes with separated predetermined nucleation for PET, Nylon 66 and PLLA at constant undercoolings and 
$n=2$. The undercoolings correspond to the temperature of the maximum crystallization rate $T_{\max }$ (indicated by the star sign) and in the vicinity of $T_{\max }$. Solid line plots illustrate influence of $A_{\mathrm{p}}(T)$ and show higher effects of the orientation at lower undercoolings. The dashed lines plots show the influence of $B_{\mathrm{p}}(T)$ which significantly reduces the influence of $A_{\mathrm{p}}(T)$ at increasing $f_{\mathrm{a}}$.

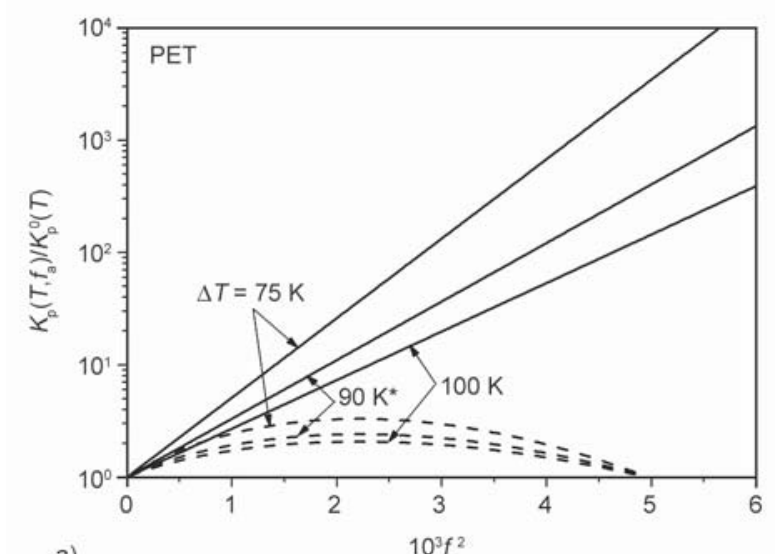

a)
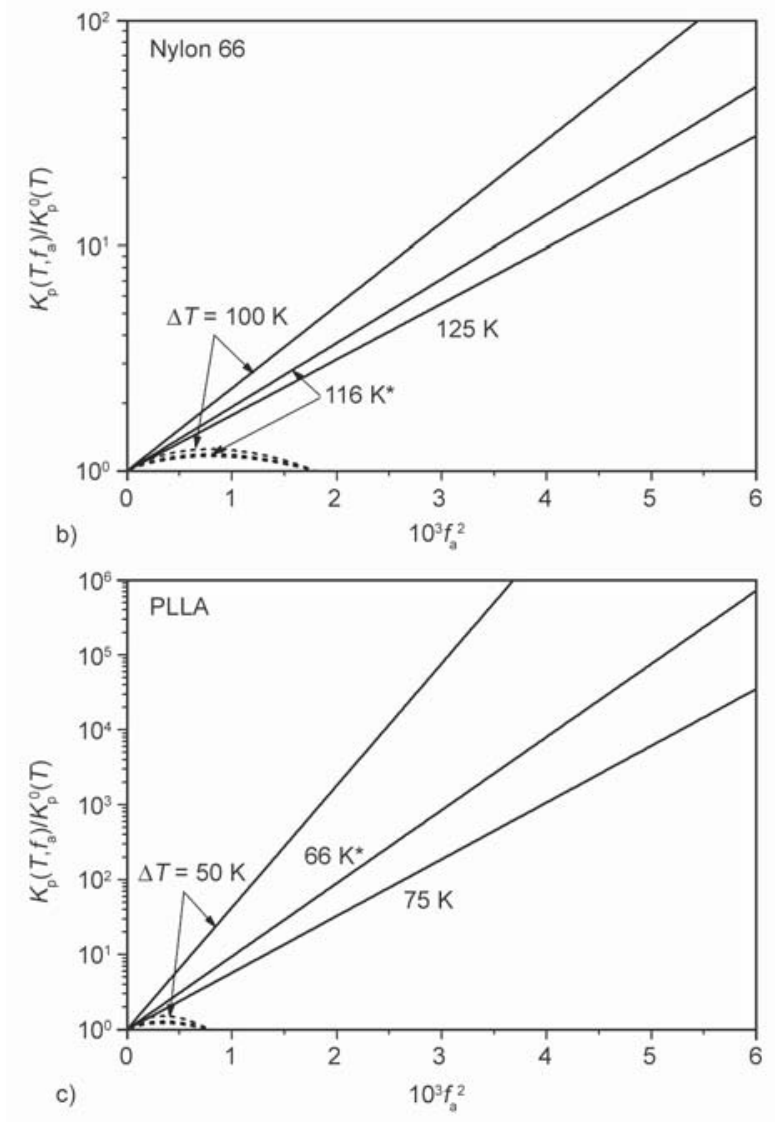

Figure 4. Reduced crystallization rate functions $K_{\mathrm{p}}\left(T, f_{\mathrm{a}}\right) / K_{\mathrm{p}}^{0}(T)$ vs. $f_{\mathrm{a}}^{2}$ computed from Equation (20) and $(29 \mathrm{a}, \mathrm{b})$ for processes with predetermined nucleation for PET (a), Nylon 66 (b) and PLLA (c) at $\Delta T$ corresponding to $T_{\max }$ (indicated by ${ }^{*}$ ) and in its vicinity. Solid lines - influence of $A_{\mathrm{p}}(T)$, dashed lines - influence of $A_{\mathrm{p}}(T)$ and $B_{\mathrm{p}}(T)$.
Maximum of the dashed-line plots in Figures 4 is a consequence of poor convergence of the series expansion. The convergence is poor at higher number of statistical segments in the chain between the entanglements approaching $N \approx 1 / f_{\mathrm{a}}$ where $N=$ $n_{\mathrm{b}} M_{\mathrm{e}} /\left(C_{\infty} m\right)$. For the tested polymers, the number $N$ of statistical segments in the elastic chain is the lowest for PET, increases respectively for Nylon 66, PLLA, iPP, and the convergence becomes poorer in this order. As reported from melt spinning experiments, the phenomenological formula limited to the first series expansion coefficient, Equation (3), adequately describes kinetics of oriented crystallization at low, as well as at high $f_{\mathrm{a}}$ values, also beyond the linear stress-orientation range, $f_{\mathrm{a}}>1$. We focus in this paper on the first expansion term, proportional to $f_{\mathrm{a}}^{2}$, with the aim to provide analytical formula for the coefficient $A(T)$ in the phenomenological Equation (3). Our analysis of the crystallization kinetics for processes with separated nucleation mechanisms allows to estimate the kinetic effects of amorphous orientation in real systems where sporadic and predetermined nucleation may coexist. Much higher values of $A_{\mathrm{s}}(T)$ calculated for sporadic nucleation in comparison with $A_{\mathrm{p}}(T)$. (Figures 3 ) indicate stronger effects of orientation $f_{\mathrm{a}}$ in the transformation kinetics at zero content of the predetermined nuclei. Such a case can be expected for polymers obtained in biosynthesis. In real systems usually both nucleation mechanism are involved and relatively high values of $A_{\mathrm{s}}$ indicate that sporadic nucleation may dominate the predetermined nucleation at higher orientations and should be considered in the overall transformation kinetics. Usually, predetermined nucleation significantly dominates sporadic nucleation under quiescent, zero-orientation conditions. Below we estimate the conditions at which involvement of sporadic nucleation is promoted by the orientation to the level of predetermined nucleation, and above.

\subsection{Rate functions at coexisting sporadic and predetermined nucleation}

In real systems, sporadic nucleation contributes to a lower or higher extent and is accounted to as accompanying predetermined nucleation (heterogeneous, memory nuclei) when considering kinetics of oriented crystallization in our approach. The presented results indicate that above a certain $f_{\mathrm{a}}$ value contribution of sporadic nucleation can dominate predetermined nucleation in the overall crystallization rate. Under 
unoriented conditions, predetermined nucleation is usually the main nucleation mechanism with a negligible contribution of sporadic nucleation. At low orientation, predetermined nucleation (if present) can be still considered as the main nucleation mechanism involved in the transformation, while sporadic nucleation contributes increasingly with increasing $f_{\mathrm{a}}$. The predetermined and sporadic nucleations are independent processes in real systems. Inverse of the crystallization half time $1 / t_{1 / 2}$ represents a frequency of achieving $1 / 2$ of the maximum degree of crystallinity. Predetermined and sporadic nucleation contribute to the global frequency the frequencies $\left(1 / t_{1 / 2}\right)_{\mathrm{p}}$ and $\left(1 / t_{1 / 2}\right)_{\mathrm{s}}$ represented by the rate function of separate sporadic and predetermined nucleation, $K_{\mathrm{p}}\left(T, f_{\mathrm{a}}\right)$, $K_{\mathrm{s}}\left(T, f_{\mathrm{a}}\right)$.

The contribution of sporadic nucleation in the global crystallization mechanism is characterized by the Avrami exponent $n$, while the component assigned to predetermined nucleation - by $n-1$. The role of sporadic nucleation mechanism at temperature $T$ and orientation $f_{\mathrm{a}}$ is characterized by the ratio of the rate functions as shown in Equation (30):

$$
\begin{aligned}
\frac{K_{\mathrm{s}}\left(T, f_{\mathrm{a}}\right)}{K_{\mathrm{p}}\left(T, f_{\mathrm{a}}\right)} & =\phi(T) \exp \left\{\left[A_{\mathrm{s}}(T)-A_{\mathrm{p}}(T)\right] f_{\mathrm{a}}^{2}+\right. \\
& \left.+\left[B_{\mathrm{s}}(T)-B_{\mathrm{p}}(T)\right] f_{\mathrm{a}}^{3}+\ldots\right\}
\end{aligned}
$$

where the exponential function characterizes the influence of sporadic nucleation relative to the influence of predetermined nucleation at $T$ and $f_{\mathrm{a}}$. The pre-exponential function $\phi(T)$ characterizes the influence of sporadic nucleation relative to predetermined one at $T$ and zero orientation (Equation (31)):

$$
\begin{gathered}
\phi(T)=\frac{K_{\mathrm{s}}^{0}(T)}{K_{\mathrm{p}}^{0}(T)}=\left\{\frac{1}{n N_{0}^{\mathrm{n} /(\mathrm{n}-1)} R_{0}^{(4-n) /(\mathrm{n}-1)}} \frac{k T N_{\mathrm{s}}}{h G_{0}} .\right. \\
\left.\cdot \exp \left[-\frac{2 c^{2} \sigma^{2} \sigma_{\mathrm{e}}}{k T\left(f \Delta h_{\mathrm{m}}\right)^{2}}\left(\frac{T_{\mathrm{m}}^{0}}{\Delta T}\right)^{2}-\frac{4 b \sigma \sigma_{\mathrm{e}}}{k T f \Delta h_{\mathrm{m}}} \frac{T_{\mathrm{m}}^{0}}{\Delta T}\right]\right\}^{1 / \mathrm{n}}
\end{gathered}
$$

where $2 \leq n \leq 4$. For one-dimensional crystal growth at sporadic or predetermined nucleation we have $n=2$ in Equation (31). At $n=4$ we have 3-dimensional growth at sporadic or predetermined nucleation. $N_{0}$ is the volume density of predetermined nuclei, $G_{0}$ the pre-exponential factor of the crystal growth rate. In our computations we assume $G_{0} \approx$ $10^{5} \mathrm{~cm} / \mathrm{s}$ [75].

Kinetic units involved in sporadic nucleation and crystal growth are identified with the chain statistical segments. Number of the kinetic units per unit volume of the amorphous phase is $N_{\mathrm{s}}=\rho_{\mathrm{a}} N_{\mathrm{A}} n_{\mathrm{b}} /\left(C_{\infty} m\right)$ where $\rho_{\mathrm{a}}$ is the amorphous phase density. For PET we have $\rho_{\mathrm{a}}(T)=\rho_{0}-\rho_{1}(T-273 \mathrm{~K})$ and for iPP, Nylon 66 and PLLA $\rho_{\mathrm{a}}(T)=\rho_{0} /\left[1+\rho_{1}(T-273 \mathrm{~K})\right]$ where $\rho_{0}$, $\rho_{1}$ coefficients are listed in Table 1 . We assume that the dimension $R_{0}$ at which the crystal growth does not proceed corresponds to the critical dimension of primary nucleus, given by Equation (32):

$R^{*}=\left(V^{*}\right)^{1 / 3}=\frac{\left(2 c^{2} \sigma^{2} \sigma_{\mathrm{e}}\right)^{1 / 3}}{f \Delta h_{\mathrm{m}}} \frac{T_{\mathrm{m}}^{0}}{\Delta T}$

Example computations of the ratio of the pre-exponential function $\phi$ vs. $\Delta T$ are presented for PET in Figures 5 and 6 . In the computations we assume $R_{0}=R^{*}$, $5 R^{*}, 10 R^{*}$ and the predetermined nuclei density $N_{0}=$ $10^{6}, 10^{7}, 10^{8} \mathrm{~cm}^{-3}$.

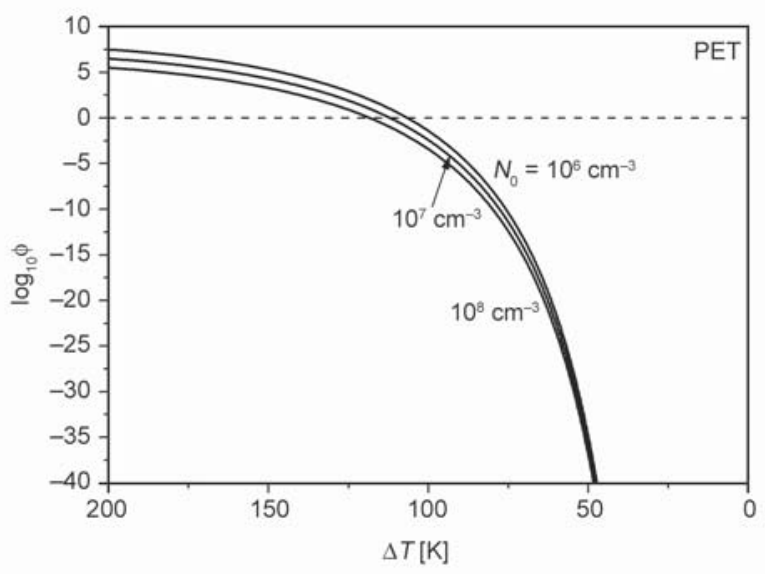

Figure 5. Ratio of the crystallization rate functions at zero orientation $\phi$ vs. $\Delta T$ computed from Equation (31) for PET at predetermined nuclei density $N_{0}=10^{6}$, $10^{7}, 10^{8} \mathrm{~cm}^{-3}, R_{0}=R^{*}, G_{0}=10^{5} \mathrm{~cm} / \mathrm{s}, n=2$

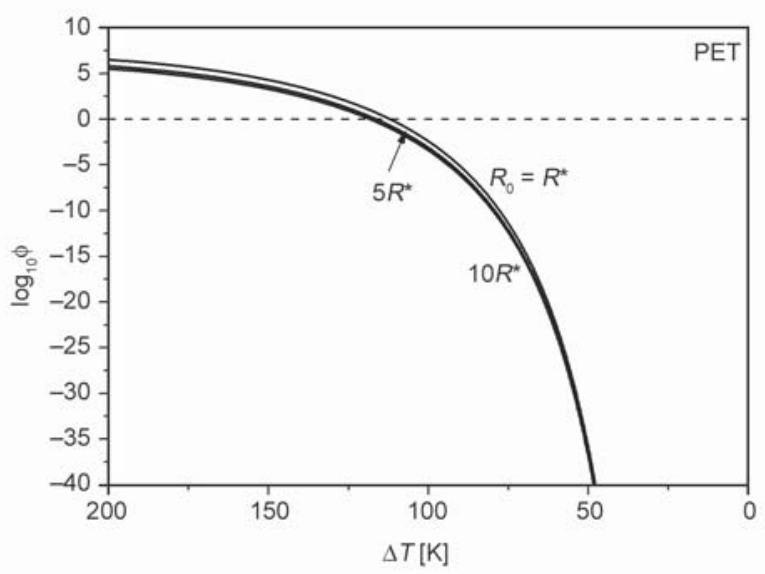

Figure 6. Ratio of the crystallization rate functions at zero orientation $\phi$ vs. $\Delta T$ computed from Equation (31) for PET at $R_{0}=R^{*}, 5 R^{*}, 10 R^{*}, N_{0}=10^{7} \mathrm{~cm}^{-3}, G_{0}=$ $10^{5} \mathrm{~cm} / \mathrm{s}, n=2$ 
Figures 5 and 6 indicate that the thermodynamic factors associated with undercooling strongly reduce the involvement of sporadic nucleation by orders of magnitude, in particular at low undercoolings. Figure 5 shows the reduction by about one order of the magnitude at increasing the content of predetermined nuclei $N_{0}$ by one order in the range $10^{6}-10^{8} \mathrm{~cm}^{-3}$. Rather marginal influence of the crystallite dimension $R_{0}$ at which the growth does not proceed is illustrated in Figure 6. An increase of $R_{0}$ in the range between the critical value $R^{*}$ and $10 R^{*}$ reduces the involvement of sporadic nucleation by about one order of the magnitude.

Very low values of the pre-exponential function $\phi$ in a wide range of undercoolings are responsible for strong reduction of the involvement of sporadic nucleation in the global transformation kinetics. But the exponential function in Equation (30) is responsible for an increase of the involvement of sporadic nucleation with increasing $f_{\mathrm{a}}$ because $A_{\mathrm{s}}(T) / A_{\mathrm{p}}(T)>1$ (Figures 3 ) and $B_{\mathrm{s}}(T) / B_{\mathrm{p}}(T)>1$. At small $f_{\mathrm{a}}$ values, the ratio $K_{\mathrm{s}}\left(T, f_{\mathrm{a}}\right) / K_{\mathrm{p}}\left(T, f_{\mathrm{a}}\right) \ll 1$ due to very low values of $\phi(T)$ and the global rate function is controlled by predetermined nucleation, $K\left(T, f_{\mathrm{a}}\right) \cong K_{\mathrm{p}}\left(T, f_{\mathrm{a}}\right)$.

Line plots in Figure 7 show the global rate function approximated by the rate function for the process controlled by predetermined nucleation $K\left(T, f_{\mathrm{a}}\right) \cong$ $K_{\mathrm{p}}\left(T, f_{\mathrm{a}}\right)=K_{0}(T) \exp \left\lfloor A_{\mathrm{p}}(T) f_{\mathrm{a}}^{2}\right\rfloor$ vs. $10^{3} f_{\mathrm{a}}$ calculated from Equation (29a) for PET at $\Delta T=165$ and $185 \mathrm{~K}$ with the assumption $A(T)=A_{\mathrm{p}}(T)$ in the range of small $f_{\mathrm{a}}$ values. The experimental points correspond to several undercooling values in the range $165-185 \mathrm{~K}$ [11]. The computed line plots and the experimental

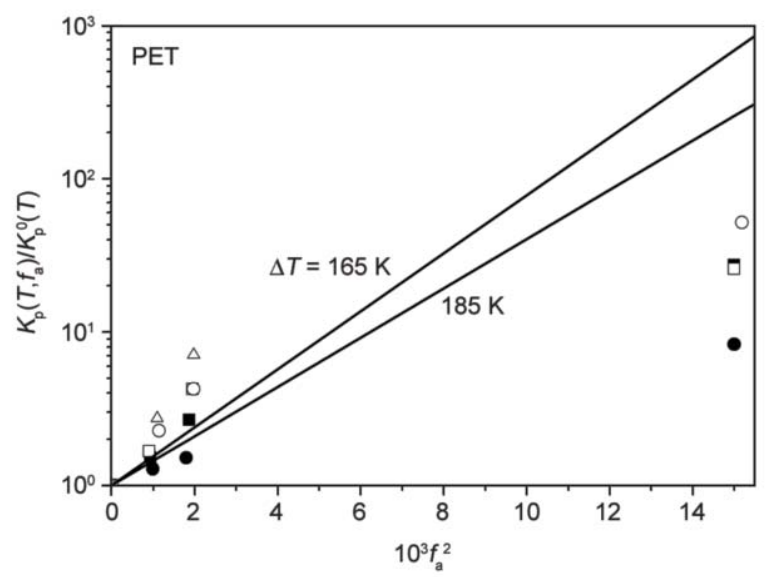

Figure 7. Reduced rate function $K\left(T, f_{\mathrm{a}}\right) / K_{0}\left(T, f_{\mathrm{a}}\right)$ vs. $10^{3} f_{\mathrm{a}}^{2}$ for PET. Experimental points [11]: $\Delta T=185 \mathrm{~K}(\bullet)$,

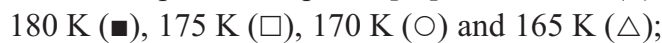
line plots: $K\left(T, f_{\mathrm{a}}\right) / K_{0}\left(T, f_{\mathrm{a}}\right)$ calculated from Equation (29a) at $\Delta T=185 \mathrm{~K}$ and $165 \mathrm{~K}$. points correspond to large undercoolings and remain within the same order of magnitude at low orientations and increase with increasing $f_{\mathrm{a}}$ and with decreasing $\Delta T$. At the highest $f_{\mathrm{a}}$ value, the experimental points depart more from the linear plots and indicate substantial non-linear effects in the oriented crystallization kinetics. The effects will be discussed in the range of non-linear stress-orientation behavior at higher tensile stresses and $f_{\mathrm{a}}$ in a separate work.

One can expect that with increasing $f_{\mathrm{a}}$, the ratio $K_{\mathrm{s}}\left(T, f_{\mathrm{a}}\right) / K_{\mathrm{p}}\left(T, f_{\mathrm{a}}\right)$ approaches unity at a certain orientation and the involvement of both nucleation mechanisms in the overall crystallization kinetics is of the same order of magnitude. Further increase of $f_{\mathrm{a}}$ should lead to domination of sporadic nucleation in the transformation kinetics. Crystallization half-time analysis of oriented crystallization in melt spinning of fibers made by Patel and coworkers $[5,27]$ indicates that in the range of high $f_{\mathrm{a}}$, the orientation effects are also satisfactorily described by the function $\exp \left\lfloor A(T) f_{\mathrm{a}}^{2}\right\rfloor$ with single phenomenological parameter $A(T)$, the same as in the range of small $f_{\mathrm{a}}$, proposed by Ziabicki $[2,47]$.

Basing on the series expansion approach, we formulate a hypothesis that the involvement of sporadic nucleation which is predicted here to increase with increasing $f_{\mathrm{a}}$ is responsible for an apparent agreement of the rate function expressed by the factor $\exp \left\lfloor A(T) f_{\mathrm{a}}^{2}\right\rfloor$ with the experimental observations at high orientation produced at high-speed melt spinning processes $[5,27]$.

\subsection{Rate functions of oriented crystallization of polymorphic structures}

The oriented crystallization coefficients of the rate functions derived in the above sections concern transformation of the oriented amorphous phase into a single crystalline structure. The coefficients derived for each of the transformation mechanism controlled by predetermined or sporadic nucleation are expressed in terms of the parameters specific to the single polymorph, such as melting heat $\Delta h_{\mathrm{m}}$, equilibrium melting point under zero stresses $T_{\mathrm{m}}^{0}$, surface free energy densities $\sigma$ and $\sigma_{\mathrm{e}}$ of the crystalline forms and thickness of the surface nucleus of crystal growth $b$. The parameters differ for different crystalline polymorphs possible to grow in the system subjected to amorphous orientation.

With the assumption that the other polymorphs do not interfere with the above parameters specified for 
each polymorph, we can consider transformation kinetics of each polymorphs in the system as independent of the other, except for the oriented amorphous source phase being common for all of them. With the parameters specific for each polymorph, the influence of amorphous orientation on the crystallization rate function for each of them expresses by the temperature-dependent oriented crystallization coefficients, Equations (28a), (29a). Important point is to specify the temperature ranges at which oriented crystallization of the polymorph proceeds, i.e. below the equilibrium melting point for the polymorph under the morphous orientation. With the ranges of crystallization temperature assigned to each of the polymorphs, the oriented crystallization rate functions of each polymorph can be calculated with the oriented crystallization coefficients expressed by Equations (28a), (29a). The progress of crystallization in the oriented systems with a specified contribution of each polymorph can be calculated from a coupled set of kinetic equations of polymorphic transformation, present in the literature [72-74] and extended to oriented crystallization.

The influence of amorphous orientation on the ranges of crystallization temperatures for particular polymorphs is illustrated in Figures 8 where Gibbs free energy $g$ is plotted schematically vs. temperature in a linear approximation near the equilibrium melting points of the polymorphs in a three-phase system composed of two crystalline polymorphs, ' 1 ' and ' 2 ', and oriented or unoriented (dashed line) amorphous phase. Entropy of the oriented amorphous phase is

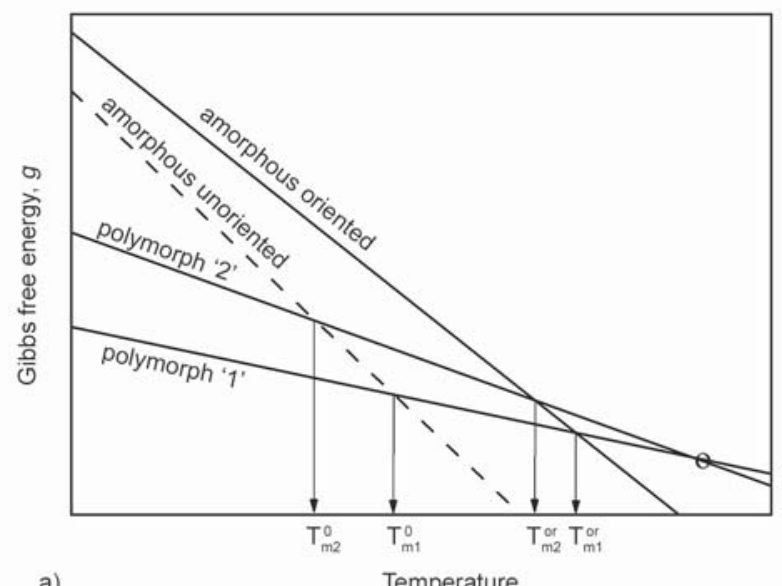

decreased by the decrease in configurational entropy of the chains under the tensile stresses and the slope of the free energy plot of oriented amorphous phase vs. temperature in Figures 8 is smaller than the slope of the unoriented one.

The tensile stresses do not influence entropy of the crystalline polymorphs to such an extent as of the configurational entropy of the amorphous phase and the slopes of the free energy plots of the polymorphs remain unchanged in this approximation. Entropies of the polymorphs differ and it is assumed that the polymorph ' 1 ' has the lower entropy in the example.

Figure 8a illustrates the behavior of the systems in which the equilibrium melting point of the lower entropy polymorphic form ' 1 ' is higher than that of polymorph ' 2 ' in the unoriented state, $T_{\mathrm{m} 1}^{0}>T_{\mathrm{m} 2}^{0}$, and Figure $8 \mathrm{~b}-$ of the systems where $T_{\mathrm{m} 1}^{0}<T_{\mathrm{m} 2}^{0}$. The order of the polymorphs melting points in the unoriented state is associated with the ratio of the melting enthalpy-to-entropy ratio, $\Delta h_{\mathrm{m}}^{0} / \Delta s_{\mathrm{m}}^{0}$, of the polymorphs. In the case of systems characterized by the free energy plots presented in Figure 8a, meta-stability of the higher enthalpy and/or higher entropy form ' 2 ' is preserved by the amorphous orientation in the entire range of crystallization temperatures. The crystallization temperature range is shifted by amorphous orientation to higher values, and the apparent thermodynamic equilibrium between the polymorphs remains above the melting points of both crystalline forms. Such situation may be representative for melt spinning of polyolefins. In the case of isotactic polypropylene, thermodynamic conditions for crystallization

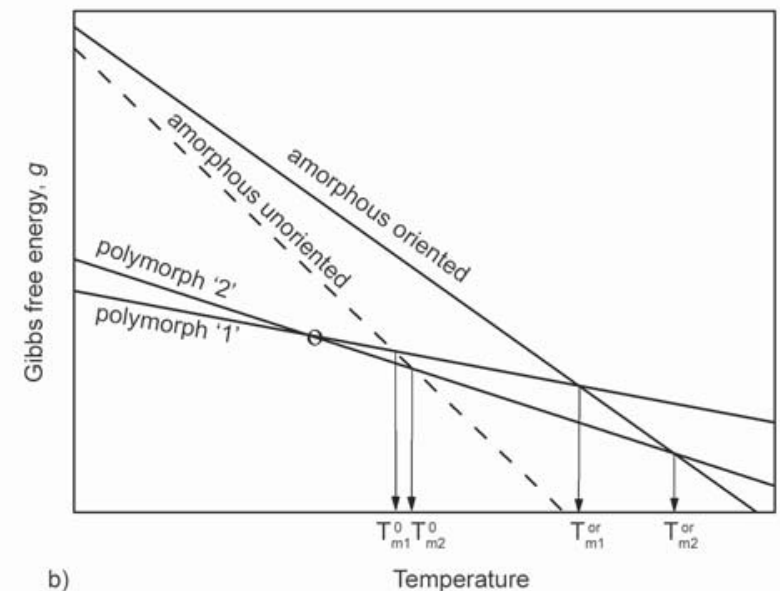

b)

Figure 8. Schematic plots of the Gibbs free energy $g$ vs. temperature in a three-phase system composed of two polymorphic crystalline forms ' 1 ', ' 2 ' and oriented or unoriented (dashed line) amorphous phase. (a) stable polymorph ' 1 ' and meta-stable polymorph ' 2 ' in the entire crystallization temperature range; (b) polymorph ' 1 ' stable at low temperatures and polymorph ' 2 ' stable at high temperatures. 
in the stable $\alpha$ and meta-stable $\beta$ forms are preserved in the entire range of crystallization temperature in unoriented and oriented systems, like in Figure 8a. Figure $8 \mathrm{~b}$ shows that at increasing melting heat of the high entropy polymorphic form ' 2 ' (free energy plot is shifted down), thermal stabilities of the polymorphs in the unoriented state are reversed at a high temperature range. The polymorphic form ' 2 ' is more stable than the polymorph ' 1 ' in the high temperature range, above the temperature of thermodynamic equilibrium of the polymorphs, and becomes a single crystalline form above the equilibrium melting point of the low entropy polymorph ' 1 '. Amorphous orientation relatively does not influence the equilibrium point between the polymorphs, but it does extend the range of thermal stability of the higher entropy polymorph and shifts the range to higher temperatures. This may concern also stabilization of mesophases by stress-induced orientation in a high temperature range during high-speed melt spinning of polyesters or polyamides, followed by transformation to a more stable crystalline form at lower temperatures.

\section{Conclusions}

Crystallization half-time analysis of oriented crystallization $[5,27]$ indicates that the orientation effects in the crystallization kinetics can be satisfactorily described by exponential function $\exp \left\lfloor A(T) f_{\mathrm{a}}^{2}\right\rfloor$ of the single quadratic term of the orientation factor $f_{\mathrm{a}}$ multiplied by the phenomenological coefficient $A(T)$. Equation (3) adequately describes kinetics of oriented crystallization at low, as well as at high $f_{\mathrm{a}}>0.1$ values beyond the linear stress-orientation range. Actually available models in the literature are not satisfactory for calculating the crystallization kinetics of polymers under variable orientation and temperature with the use of the $\exp \left\lfloor A(T) f_{\mathrm{a}}^{2}\right\rfloor$ formula due to the lack of a general expression for the function $A(T)$. In the series expansion approach to the oriented crystallization kinetics we focus on the first expansion term proportional to $f_{\mathrm{a}}^{2}$ with the aim to derive formula for the temperature-dependent coefficient $A(T)$ basing on the Hoffman-Lauritzen kinetic theory of crystal nucleation and growth. Our calculation are based on the 'proportional expansion' approach [46] in the steady-state limit. The rate functions of oriented crystallization with separated sporadic and predetermined nucleation mechanisms are calculated, as well as their involvement in real systems subjected to orienting stresses. The crystallization free energy accounts for the entropy of uniaxial orientation of the amorphous chains. The orientation entropy is expanded in the power series over $f_{\mathrm{a}}$ and the expansion coefficients are calculated for Gaussian chains in the linear stress-orientation range.

Example computations of the oriented crystallization coefficient $A_{\mathrm{p}}(T)$ for crystallization with separate predetermined nucleation and the ratio $A_{\mathrm{s}}(T) / A_{\mathrm{p}}(T)$ of the coefficients for processes with separated sporadic and predetermined nucleation are presented for iPP, PET, Nylon 66 and PLLA. High positive values of the coefficient are predicted in the entire range of undercooling. The $A_{\mathrm{p}}$ values increase from several hundred at large undercoolings to several thousand at decreasing the undercooling to $30 \mathrm{~K}$. The Avrami exponent $n$ has no influence on $A_{\mathrm{p}}$. The predicted values of $A_{\mathrm{p}}$ for PET are of the order of the experimental values of the oriented crystallization coefficient $A$ determined for Equation (3) at several temperatures [11], and both values increase with increasing the temperature.

The coefficient $A_{\mathrm{s}}(T)$ for processes with separated sporadic nucleation is higher by a factor of about 4 up to 7 at larger undercoolings and increases at approaching the equilibrium melting temperature $T_{\mathrm{m}}^{0}$. Higher values of $A_{\mathrm{s}}$, in comparison to $A_{\mathrm{p}}$, result from higher influence of orientation entropy on the free energy barrier of sporadic nucleation. Higher Avrami exponents only slightly reduce $A_{\mathrm{s}}$. Relatively high $A_{\mathrm{s}}$ values indicate that sporadic nucleation should be considered in the overall transformation kinetics at higher orientations. Example computations of the crystallization rate functions for PET, Nylon 66 and PLLA with separated nucleation mechanisms indicate higher effects of amorphous orientation at lower undercoolings.

With the aim to estimate an involvement of sporadic and predetermined nucleation in real systems in the overall crystallization kinetics, followed by crystal growth, we discuss the ratio of the oriented crystallization rate functions calculated as independent kinetic characteristics. The conditions at which the involvement of sporadic nucleation approaches the level of the predetermined one in the kinetics are formulated. The involvement of sporadic nucleation in the global kinetics, relative to the predetermined one, is characterized by a product of an exponential function responsible for the orientation effects and 
a pre-exponential factor $\phi(T)$ which characterizes relative contribution of the nucleation mechanism under zero orientation, Equations (30) and (31).

At low orientation, the main crystallization mechanism is associated with predetermined nucleation and the overall crystallization rate is controlled by the predetermined nucleation, and $A(T) \cong A_{\mathrm{p}}(T)$. The relative involvement of sporadic nucleation at small orientations is reduced by many orders of the magnitude by thermodynamic factors associated with the undercooling.

Reduction of the predetermined nuclei content $N_{0}$ by one order of the magnitude results in an increase of the involvement of sporadic nucleation by one order. Marginal influence of the crystallite dimension $R_{0}$ at which the crystal growth does not proceed is predicted. An increase of $R_{0}$ between the critical value $R^{*}$ and $10 R^{*}$ reduces the sporadic nucleation involvement by one order of the magnitude.

Example computations of the crystallization rate function at small orientations for PET approximated by the rate function controlled by predetermined nucleation are in qualitative agreement with the experimental results [11] and illustrate influence of the undercooling. We conclude that with increasing amorphous orientation, involvement of both nucleation mechanisms may approach the same order at some orientation level and further increase of $f_{\mathrm{a}}$ can lead to domination of sporadic nucleation. Basing on the series expansion approach we formulate a hypothesis that the increase of sporadic nucleation rate at increasing $f_{\mathrm{a}}$ is responsible for an apparent agreement of the experimental observations [5, 27] of the crystallization kinetics at high amorphous orientation with the single-term $\exp \left\lfloor A(T) f_{\mathrm{a}}^{2}\right\rfloor$ formula.

Basing on the approach presented in this paper, the oriented crystallization rate functions can be formulated for all thermodynamically admissible transformations between pairs of phases in polymorphic systems subjected to tensile stresses, to be used in a set of kinetic equations of individual transformations between the phases in the system.

\section{Acknowledgements}

This research was supported by the basic funds of the Institute of Fundamental Technological Research, Polish Academy of Sciences for scientific research.

\section{References}

[1] Dumbleton J. H.: Spin orientation measurement in polyethylene terephthalate. Textile Research Journal, 40, 1035-1041 (1970). https://doi.org/10.1177/004051757004001111

[2] Ziabicki A.: Fundamentals of fiber formation.Wiley, London (1976).

[3] Ziabicki A., Kawai H.: High-speed fiber spinning. Wiley, New York (1985).

[4] Zieminski K. F., Spruiell J. E.: On-line studies and computer simulation of the melt spinning of nylon-66 filaments. Journal of Applied Polymer Science, 35, 22232245 (1988).

https://doi.org/10.1002/app.1988.070350822

[5] Patel M., Spruiell J. E.: Crystallization kinetics during polymer processing - Analysis of available approaches for process modeling. Polymer Engineering and Science, 31, 730-738 (1991).

https://doi.org/10.1002/pen.760311008

[6] Ziabicki A., Jarecki L., Wasiak A.: Dynamic modelling of melt spinning. Computational and Theoretical Polymer Science, 8, 143-157 (1998).

https://doi.org/10.1016/S1089-3156(98)00028-2

[7] Spruiell J. E.: Structure formation during melt spinning. in 'Structure formation in polymeric fibers' (ed.: Salem D. R.) Hanser, Munich, 5-93 (2000).

[8] Treloar L. R. G.: Crystallisation phenomena in raw rubber. Transactions of the Faraday Socciety, 37, 84-97 (1941).

https://doi.org/10.1039/TF9413700084

[9] Smith F. S., Steward R. D.: The crystallization of oriented poly(ethylene terephthalate). Polymer, 15, 283 286 (1974). https://doi.org/10.1016/0032-3861(74)90125-6

[10] Stein R. S.: Optical studies of the stress-induced crystallization of polymers. Polymer Engineering and Science, 16, 152-157 (1976). https://doi.org/10.1002/pen.760160306

[11] Alfonso G. C., Verdona M. P., Wasiak A.: Crystallization kinetics of oriented poly(ethylene terephthalate) from the glassy state. Polymer, 19, 711-716 (1978). https://doi.org/10.1016/0032-3861(78)90128-3

[12] Bragato G., Gianotti G.: High speed spinning of poly (ethylene terephthalate) - II: Orientation induced mechanism of cold crystallization in pre-orientated yarns. European Polymer Journal, 19, 803-809 (1983). https://doi.org/10.1016/0014-3057(83)90150-7

[13] Desai P., Abhiraman A. S.: Crystallization in oriented poly(ethylene terephthalate) fibers. I. Fundamental aspects. Journal of Polymer Science Part B: Polymer Phisics, 23, 653-674 (1985).

https://doi.org/10.1002/pol.1985.180230404

[14] Shimizu J., Okui N., Kikutani T.: Fine structure and physical properties of fibers melt-spun at high speeds from various polymers. in 'High-speed fiber spinning' (eds.: Ziabicki A., Kawai H.) Wiley, New York, 429483 (1985). 
[15] Saijo K., Zhu Y-P., Hashimoto T., Wasiak A., Brzostowski N.: Oriented crystallization of crosslinked cis1,4-polybutadiene rubber. Journal of Applied Polymer Science, 105, 137-157 (2007).

https://doi.org/10.1002/app.26019

[16] Flory P. J.: Thermodynamics of crystallization in high polymers. I. Crystallization induced by stretching. Journal of Chemical Physics, 15, 397-408 (1947).

https://doi.org/10.1063/1.1746537

[17] Smith K. J., Greene A., Ciferri A: Crystallization under stress and non-Gaussian behavior of macromolecular networks. Rubber Chemistry and Technology, 39, 685711 (1966).

https://doi.org/10.5254/1.3544874

[18] Krigbaum W. R., Roe R-J.: Diffraction study of crystallite orientation in a stretched polychloroprene vulcanizate. Journal of Polymer Science Part A: Polymer Chemistry, 2, 4391-4414 (1964).

https://doi.org/10.1002/pol.1964.100021010

[19] Kobayashi K., Nagasawa T.: Crystallization of sheared polymer melts. Journal of Macromolecular Science, Part B: Physics, 4, 331-345 (1970).

https://doi.org/10.1080/00222347008212506

[20] Yamamoto M., White J. L.: Theory of deformation and strain-induced crystallization of an elastomeric network polymer. Journal of Polymer Science Part B: Polymer Physics, 9, 1399-1455 (1971).

https://doi.org/10.1002/pol.1971.160090804

[21] Gaylord R. J.: Orientation of crystallites formed in stretched polymeric networks. Journal of Polymer Science Part C: Polymer Letters, 13, 337-240 (1975). https://doi.org/10.1002/pol.1975.130130604

[22] Ziabicki A., Jarecki L.: Theoretical analysis of oriented and non-isothermal crystallization III. Kinetics of crystal orientation. Colloid and Polymer Science, 256, 332342 (1978).

https://doi.org/10.1007/BF01544326

[23] Jarecki L., Ziabicki A.: Thermodynamically controlled crystal orientation in stressed polymers. in 'Flow-induced crystallization in polymer systems' (ed.: Miller R. L.) Gordon and Breach, New York, 319-330 (1979).

[24] Katayama K., Yoon M. G.: Polymer crystallization in melt spinning: Mathematical simulation. in 'High speed fiber spinning' (ed.: Ziabicki A., Kawai H.) Wiley, New York, 207-223 (1985).

[25] Ziabicki A., Jarecki L.: The theory of molecular orientation and oriented crystallization in high-speed spinning. in 'High Speed Fiber Spinning' (ed.: Ziabicki A., Kawai H.) Wiley, New York, 225-269 (1985).

[26] Heuvel H. M., Huisman R.: Effect of winding speed on the physical structure of as-spun poly(ethylene terephthalate) fibers, including orientation-induced crystallization. Journal of Applied Polymer Science, 22, 22292243 (1978).

https://doi.org/10.1002/app.1978.070220815
[27] Patel R. M., Bheda J. H., Spruiell J. E.: Dynamics and structure development during high-speed melt spinning of nylon 6. II. Mathematical modeling. Journal of Applied Polymer Science, 42, 1671-1682 (1991). https://doi.org/10.1002/app.1991.070420622

[28] Ziabicki A., Jarecki L.: Crystallization-controlled limitations of melt spinning. Journal of Applied Polymer Science, 105, 215-223 (2007).

https://doi.org/10.1002/app.26121

[29] Krieger I. M., Dougherty T. J.: A mechanism for nonnewtonian flow in suspensions of rigid spheres. Journal of Rheology, 3, 137-152 (1959). https://doi.org/10.1122/1.548848

[30] Spruiell J. E., White J.: Structure development during the melt spinning of fibers. Applied Polymer Symposia, 27, 121-157 (1975).

[31] Kulkarni J. A., Beris A. N.: A model for the necking phenomenon in high-speed fiber spinning based on flow-induced crystallization. Journal of Rheology, 42, 971-994 (1998). https://doi.org/10.1122/1.550913

[32] Lin Y. G., Mallin D. T., Chien J. C. W., Winter H. H.: Dynamic mechanical measurements of crystallization induced gelation in thermoplastic elastomeric poly(propylene). Macromolecules, 24, 850-854 (1991).

https://doi.org/10.1021/ma00004a006

[33] Titomanlio G., Speranza V., Brucato V.: On the simulation of thermoplastic injection molding process. International Polymer Processing, 12, 45-53 (1997). https://doi.org/10.3139/217.970045

[34] Pantani R., Speranza V., Titomanlio G.: Relevance of crystallisation kinetics in the simulation of the injection molding process. International Polymer Processing, 16, 61-71 (2001). https://doi.org/10.3139/217.1620

[35] Floudas G., Hilliou L., Lellinger D., Alig I.: Shear-induced crystallization of poly( $\varepsilon$-caprolactone). 2. Evolution of birefringence and dichroism. Macromolecules, 33, 6466-6472 (2000).

https://doi.org/10.1021/ma000243b

[36] Doufas A. K., McHugh A. J., Miller C., Immaneni A.: Simulation of melt spinning including flow-induced crystallization: Part II. Quantitative comparisons with industrial spinline data. Journal of Non-Newtonian Fluid Mechanics, 92, 81-103 (2000). https://doi.org/10.1016/S0377-0257(00)00089-6

[37] Tanner R. I.: A suspension model for low shear rate polymer solidification. Journal of Non-Newtonian Fluid Mechanics, 102, 397-408 (2002). https://doi.org/10.1016/S0377-0257(01)00189-6

[38] Ziabicki A., Jarecki L., Sorrentino A.: The role of flowinduced crystallisation in melt spinning. e-Polymers, no. 072 (2004).

https://doi.org/10.1515/epoly.2004.4.1.823 
[39] Lee J. S., Shin D. M., Yung H. W., Hyun J. C.: Transient solutions of the dynamics in low-speed fiber spinning process accompanied by flow-induced crystallization. Journal of Non-Newtonian Fluid Mechanics, 130, 110 116 (2005).

https://doi.org/10.1016/j.jnnfm.2005.08.004

[40] Pantani R., Speranza V., Titomanlio G.: Simultaneous morphological and rheological measurements on polypropylene: Effect of crystallinity on viscoelastic parameters. Journal of Rheology, 59, 377-390 (2015).

https://doi.org/10.1122/1.4906121

[41] Jarecki L., Ziabicki A.: Mathematical modeling of pneumatic melt spinning of isotactic polypropylene. Part II. Dynamic model of melt blowing. Fibers and Textiles in Eastern Europe, 16, 17-24 (2008).

[42] Jarecki L., Ziabicki A., Lewandowski Z., Blim A.: Dynamics of air drawing in the melt blowing of nonwovens from isotactic polypropylene by computer modeling. Journal of Applied Polymer Science, 119, 53-65 (2011). https://doi.org/10.1002/app.31973

[43] Jarecki L., Blonski S., Blim A., Zachara A.: Modeling of pneumatic melt spinning processes. Journal of Applied Polymer Science, 125, 4402-4415 (2012).

https://doi.org/10.1002/app.36575

[44] Jarecki L., Blonski S., Zachara A.: Modeling of pneumatic melt drawing of poly-L-lactide fibers in the Laval nozzle. Industrial Engineering and Chemistry Research, 54, 10796-10810 (2015). https://doi.org/10.1021/acs.iecr.5b02375

[45] Nakamura K., Watanabe T., Katayama K., Amano T.: Some aspects of nonisothermal crystallization of polymers. I. Relationship between crystallization temperature, crystallinity, and cooling conditions. Journal of Applied Polymer Science, 16, 1077-1091 (1972). https://doi.org/10.1002/app.1972.070160503

[46] Ziabicki A.: Theoretical analysis of oriented and nonisothermal crystallization. II. Extension of the Kolmogoroff-Avrami-Evans theory onto processes with variable rates and mechanisms. Colloid and Polymer Science, 252, 433-447 (1974). https://doi.org/10.1007/BF01554749

[47] Ziabicki A: Theoretical analysis of oriented and non isothermal crystallization. I. Phenomenological considerations. Isothermal crystallization accompanied by simultaneous orientation or disorientation. Colloid and Polymer Science, 252, 207-221 (1974).

https://doi.org/10.1007/BF01638101

[48] Fisher J. C., Hollomon J. H., Turnbull D.: Nucleation. Journal of Applied Physics, 19, 775-784 (1948). https://doi.org/10.1063/1.1698202

[49] Ziabicki A.: Generalized theory of nucleation kinetics. II. Athermal nucleation involving spherical clusters. Journal of Chemical Physics, 48, 4374-4380 (1968). https://doi.org/10.1063/1.1668003

[50] Kashchiev D.: Nucleation. Butterworth-Heinemann, Oxford (2000).
[51] Khana Y. P., Reimschuessel A. C.: Memory effects in polymers. I. Orientational memory in the molten state; Its relationship to polymer structure and influence on recrystallization rate and morphology. Journal of Applied Polymer Science, 35, 2259-2268 (1988).

https://doi.org/10.1002/app.1988.070350824

[52] Fillon B., Wittman J. C., Lotz B., Thierry A.: Self-nucleation and recrystallization of isotactic polypropylene ( $\alpha$ phase) investigated by differential scanning calorimetry. Journal of Polymer Science Part B: Polymer Physics, 31, 1383-1393 (1993). https://doi.org/10.1002/polb.1993.090311013

[53] Alfonso G. C., Scardigli P.: Melt memory effects in polymer crystallization. Macromolecular Symposia, 118, 323-328 (1997).

https://doi.org/10.1002/masy.19971180143

[54] Supaphol P., Spruiell J. E.: Crystalline memory effects in isothermal crystallization of syndiotactic polypropylene. Journal of Applied Polymer Science, 75, 337-346 (2000). https://doi.org/10.1002/(SICI)10974628(20000118)75:3<337::AID-APP1>3.0.CO;2-4

[55] Ziabicki A., Misztal-Faraj B., Jarecki L.: Kinetic model of non-isothermal crystal nucleation with transient and athermal effects. Journal of Materials Science, 51, 89358952 (2016).

https://doi.org/10.1007/s10853-016-0145-8

[56] Ziabicki A.: Orientation mechanisms in the development of high-performance fibers. in 'Orientational phenomena in polymers' (eds.: Myasnikova L., Marikhin V. A.) Steinkopff, Frankfurt, Vol 92, 1-7 (1993). https://doi.org/10.1007/BFb0115443

[57] Ziabicki A., Jarecki L., Schoene A.: Transient biaxial orientation of flexible polymer chains in a wide range of deformation conditions. Polymer, 45, 5735-5742 (2004).

https://doi.org/10.1016/j.polymer.2004.05.070

[58] Ziabicki A., Alfonso G. C.: Memory effects in isothermal crystallization. I. Theory. Colloid and Polymer Science, 272, 1027-1042 (1994).

https://doi.org/10.1007/BF00652372

[59] Alfonso G. C., Ziabicki A.: Memory effects in isothermal crystallization II. Isotactic polypropylene. Colloid and Polymer Science, 273, 317-323 (1995).

https://doi.org/10.1007/BF00652344

[60] Schoene A., Ziabicki A., Jarecki L.: Transient uniaxial orientation of flexible polymer chains in a wide range of elongation rates. Polymer, 46, 3927-3935 (2005). https://doi.org/10.1016/j.polymer.2005.02.109

[61] Jarecki L.: Kinetic theory of crystal nucleation under transient molecular orientation. in 'Progress in understanding of polymer crystallization' (eds.: Reiter G., Strobl G. R.) Springer, Berlin, 65-86 (2007). https://doi.org/10.1007/3-540-47307-6_ 4

[62] George H. H.: Spinline crystallization of polyethylene terephthalate. in 'High speed fiber spinning' (eds.: Ziabicki A., Kawai H.) Wiley, New York, 271-294 (1985). 
[63] Hoffman J. D., Davis G. T., Lauritzen J. I. Jr.: The rate of crystallization of linear polymers with chain folding. in 'Treatise on solid state chemistry' (ed.: Hannay N. B.) Springer, Boston, 497-614 (1976). https://doi.org/10.1007/978-1-4684-2664-9 7

[64] Ziabicki A., Jarecki L.: Non-linear theory of stress-optical relations in polymer fluids. in 'Progress and trends in rheology II' (eds.: Giesekus H., Hibberd M. F.) Springer, Heidelberg, 83-86 (1988). https://doi.org/10.1007/978-3-642-49337-9 18

[65] Salem D. R.: Draw-induced structure development in flexible-chain polymers. in 'Structure formation in polymeric fibers' (ed.: Salem D. R.) Hanser, Munich, 118 184 (2001).

[66] Jarecki L., Misztal-Faraj B.: Non-linear stress-orientation behavior of flexible chain polymers under fast elongational flow. European Polymer Journal, 95, 368381 (2017).

https://doi.org/10.1016/j.eurpolymj.2017.08.028

[67] Pȩcherski R. B.: Finite deformation plasticity with strain induced anisotropy and shear banding. Journal of Materials Processing Technology, 60, 35-44 (1996). https://doi.org/10.1016/0924-0136(96)02305-9

[68] Pieczyska E. A., Pecherski R. B., Gadaj S. P., Nowacki W. K., Nowak Z., Matyjewski M.: Experimental and theoretical investigations of glass-fibre reinforced composite subjected to uniaxial compression for wide spectrum of strain rates. Archive of Mechanics, 58, 273-291 (2006).

[69] Jarecki L., Ziabicki A.: Thermodynamically controlled crystal orientation in stressed polymers: 1 . Effects of strain energy of crystals embedded in an uncrosslinked amorphous matrix and hydrodynamic potential. Polymer, 18, 1015-1021 (1997).

https://doi.org/10.1016/0032-3861(77)90005-2
[70] Jarecki L.: Thermodynamics of deformation of an isolated polymer chain. Colloid and Polymer Science, 257, 711-719 (1979). https://doi.org/10.1007/BF01474099

[71] Jarecki L., Ziabicki A.: Development of molecular orientation and stress in biaxially deformed polymers. I. Affine deformation in a solid state. Polymer, 43, 25492559 (2002).

https://doi.org/10.1016/S0032-3861(02)00031-9

[72] Ziabicki A., Misztal-Faraj B.: Modeling of phase transitions in three-phase polymorphic systems: Part I. Basic equations and example simulation. Journal of Materials Research, 26, 1586-1595 (2011). https://doi.org/10.1557/jmr.2011.195

[73] Misztal-Faraj B., Ziabicki A.: Modeling of phase transitions in three-phase polymorphic systems: Part II. Effects of material characteristics on transition rates. Journal of Materials Research, 26, 1596-1604 (2011). https://doi.org/10.1557/jmr.2011.196

[74] Coccorullo I., Pantani R., Titomanlio G.: Crystallization kinetics and solidified structure in iPP under high cooling rates. Polymer, 44, 307-318 (2003). https://doi.org/10.1016/S0032-3861(02)00762-0

[75] Janeschitz-Kriegl H.: Crystallization modalities in polymer melt processing. Fundamental aspects of structure formation. Springer, Wien (2010). https://doi.org/10.1007/978-3-211-87627-5 